Zuerst ersch. in: Psychologie - Kultur - Gesellschaft / B. Mayer \& H.-J. Kornadt (Hrsg). Wiesbaden: VS, 2010, S. 273-312

\title{
Gegenseitige Bereicherung psychologischer und sozial- und wirtschaftswissenschaftlicher Forschung. Eine kulturvergleichende Perspektive
}

\section{Gisela Trommsdorff}

\section{Einführung}

Was macht die Psychologie für die Sozial- und Wirtschaftswissenschaften und umgekehrt, was macht die Sozialwissenschaften für die Psychologie so interessant? Diese Frage ist nicht so zu verstehen, dass „die Psychologie“" oder „die Sozial- und Wirtschaftswissenschaften" jeweils einheitliche Wissenschaften wären. Vielmehr gliedem sie sich in verschiedene Teildisziplinen und Ansätze. Ich kenne jedoch keine soziologische Theorie, die nicht in irgendeiner Weise wenigstens implizit Bezug auf individuelles Handeln und zugrunde liegende mentale Prozesse nimmt und damit in die Nähe psychologischer kulturinformierter Forschung rücken könnte. Dass in der Soziologie die mentalen Prozesse und in der Psychologie die soziologischen Erkenntnisse allerdings nur implizit oder kursorisch berücksichtigt werden, kann man als eine Aufforderung an die Psychologie und die Sozial- und Wirtschaftswissenschaften verstehen, ihren möglichen Beitrag für die jeweils andere Wissenschaft zu erkennen und zu nutzen.

Ein bekanntes Beispiel für die Nähe einer höchst eintlussreichen soziologischen Theorie zur Psychologie ist Max Webers (1988) religionssoziologische Analyse des Kapitalismus und seine Annahme, dass der freiwillige Verzicht auf sofortige Bedürfnisbefriedigung zugunsten der Investition dadurch gewonnener Ressourcen in zukünftige höherwertige Belohnungen die Grundlage für späteren wirtschaftlichen Erfolg sei. Diese Annahme liegt auch dem in der Psychologie einflussreichen Paradigma für den Belohnungsaufschub als Bedingung für erfolgreiches Handeln zugrunde (Mischel, 1996; Mischel \& Ayduk, 2004). Was sind die Bedingungen für die Verzichtbereitschaft unmittelbarer zugunsten späterer Belohnungen? Aus psychologischer Sicht werden hier Prozesse der Selbstregulation wirksam. Diese können interindividuell u.a. je nach Sozialisationsbedingungen, intermalisierten Werten, Zukunftsorientierung, Selbstkonzept und situativen Anregungsbedingungen variieren. Aber solche psychologischen Er-

Die Arbeit entstand teilweise im Zusammenhang mit a) dem DFG tinanzierten Projekt "Value of Children in Six Cultures" (DFG, AZ: ir $169 / 9-1,2,3$ ) sowie mit b) dem Teilprojekt der DFG-finanzierten Forschergruppe „Grenzen der Absichtlichkeit”": „Entwicklungsbedingungen von Absichtlichkeit und ihrer Grenzen" (DFG GZ, TR 169/14-2).

Konstanzer Online-Publikations-System (KOPS)

URN: http://nbn-resolving.de/urn:nbn:de:bsz:352-opus-106812

URL: http://kops.ub.uni-konstanz.de/volltexte/2010/10681/ 
kenntnisse alleine greifen für die Analyse erfolgreichen Wirtschaftens zu kurz wenn soziale Strukturen, spezifische Konstellationen kultureller Orientierungen und deren historische Entwicklung nicht mit berücksichtigt werden. Und sind dafür nicht wiederum spezifische sozialwissenschaftliche Ansätze notwendig?

Andere Beispiele soziologischer Theorien mit unübersehbaren Implikationen für psychologische Fragestellungen sind Durkheims (1933) Theorien zu Bedingungen von Anomie, abweichendem Verhalten, gesellschaftlicher Desintegration. Inzwischen sind die Sozialwissenschaften in der Folge von Weber, Durkheim, Parsons und Merton hinsichtlich großer soziologischer Theorien sehr zurückhaltend geworden. Heute werden Theorien begrenzter Reichweite bevorzugt. Das heißt jedoch nicht, dass psychologische Phänomene in den neuen Ansätzen an Bedeutung verloren haben, auch wenn diese Bedeutung meist nur implizit sichtbar wird.

Wenn in makrosoziologischen Ansätzen der Fokus auf gesamtgesellschaftlichen und strukturellen Phänomenen liegt, erscheint allerdings die Bedeutung des individuellen Akteurs relativ unerheblich. Ein Beispiel einer auf einen begrenzten Gegenstandsbereich bezogenen soziologischen Theorie sind die Arbeiten von Ralf Dahrendorf (1957, 1965). Dahrendorf (1957) betont Prozesse der Institutionalisierung von Klassenkonflikten, wodurch anhaltend bestehende Interessenkonflikte gewaltlos ausgehandelt werden können. Der Ausgangspunkt seiner Theoriebildung sind gesellschaftliche Systeme und nicht individuelle Akteure.

Wenn der Ausgangspunkt seiner Theorie individuelle Akteure wären, müssten psychologische Ansätze herangezogen werden, um z.B. die Frage zu untersuchen, unter welchen Bedingungen die individuellen Akteure ausreichend Legitimität der Institution für deren Handeln zuschreiben, d.h. auch bereit sind, dem Kollektiv (der Institution) Kompetenz und Verantwortung für Entscheidungen zu attribuieren und die Ziele sowie die Entscheidungen des Kollektivs zu akzeptieren. „Klassen“ handeln nicht; und ,aushandeln“ können nur Individuen. Individuelle Akteure agieren nicht unabhängig voneinander und nicht unabhängig von gegebenen sozio-strukturellen Faktoren. Sie verändern die Ziele und das Verhalten des Kollektivs (der Institution). Individuelles und kollektives Handeln wird jeweils durch unterschiedliche Bedingungen beeinflusst und ist durch verschiedene Theorieansätze zu erklären. Phänomene wie kollektive Identität und kollektive Intentionalität können jeweils auf Grundlage psychologischer sowie auch sozialwissenschaftlicher Theorien und Methoden untersucht werden, denn sie sind jeweils ein Ergebnis psychologischer und soziologischer Prozesse. Daher werden jeweils entsprechend verschiedene Forschungsfragen zugrunde gelegt.

Auch Institutionen und ihre Legitimationsgrundlagen sind selbst ein Ergebnis komplexer psychologischer sowie sozio-kultureller und ökonomischer Prozesse. Die Legitimität von Institutionen hat wiederum Implikationen für makrosozio- logische (z.B. Wirtschafts- und Sozialstruktur, Arbeitsmarkt, Wirtschaftswachstum, Armutsrisiko, Bildungsniveau) und mikrosoziologische Phänomene (z.B. individuelles Konsum-, Gesundheits-, Bildungs- und Wahlverhalten). Damit werden auch psychologische Fragen von Vertrauen, Sicherheitsbedürfnis und Risikobereitschaft angesprochen. Zum Beispiel wäre theoretisch und praktisch relevant. ob bei hoher Legitimität von Institutionen die erlebte individuelle Sicherheit und dadurch vermittelt der Anreiz für langfristige Investitionen (z.B. Umweltschutz) in kollektive Güter (Gemeinschaftsgüter) oder die Bereitschaft für ehrenamtliche Tätigkeiten und soziales Engagement eher erhöht werden. Diese Beispiele mögen verdeutlichen, dass die Untersuchung gesellschaftlicher Phänomene gleichzeitig für verschiedene Disziplinen relevant sein kann. Psychologische, soziologische oder ökonomische Erklärungsansätze alleine greifen jeweils zu kurz.

Im Folgenden soll zunächst gefragt werden, worin spezifische Beiträge psychologischer Forschung zur Klärung sozialer Phänomene bestehen können. Dabei sollen die Möglichkeiten und Grenzen psychologischer Ansätze dargelegt werden. Sodann wird beispielhaft aus der Sicht der Sozial- und Wirtschaftswissenschaften gezeigt, welche Ergebnisse (vor allem kulturinformierter) psychologischer Forschung dort fruchtbar geworden sind. Abschließend wird auf einige theoretische und methodologische Aspekte der unterschiedlichen Analyseebenen der psychologischen und der sozialwissenschaftlichen Forschung eingegangen, und es werden Möglichkeiten für deren Verknüpfung diskutiert.

\section{Möglichkeiten und Grenzen eines Beitrages der Psychologie zur Aufklärung sozialer Phänomene}

Kann (und will) die Psychologie einen Beitrag zur sozialwissenschaftlichen Forschung leisten? Soweit sich die Psychologie naturwissenschaftlicher Methoden bedient und in experimentellen Studien den sozialen Kontext möglichst als Störvariable auszublenden versucht, kann man ihren Beitrag zur Aufklärung sozialer Phänomene bezweifeln, jedenfalls wenn sich die Psychologie als eine kontextfreie Wissenschaft versteht.

\subsection{Psychologie als kontextfreie Wissenschaft vom individuellen Handeln?}

In soziologischen Theorien werden, wie gesagt, selten individuelle mentale Prozesse explizit berücksichtigt, und, wie gesagt, in psychologischen Theorieansätzen wird häufig der sozio-kulturelle und ökonomische Kontext ignoriert. Beide Einseitigkeiten können nur sehr begrenzt zur Aufklärung sozialer Phänomene 
beitragen. In der psychologischen Grundlagenforschung ist ein solcher direkter Beitrag zur Aufklärung sozialer Phänomene eher nicht beabsichtigt; er liegt eher nahe in den Bereichen der angewandten Psychologie (z.B. der Arbeits- und Organisations-, der Rechts- und Gesundheitspsychologie). Die allgemeine und experimentelle Psychologie vernneidet explizit, den sozialen Kontext mit einzubeziehen, weil dies zur Konfundierung von Variablen führen würde, was keine generalisierbaren Aussagen ermöglicht. Auch die experimentelle Sozialpsychologie versucht in Laboruntersuchungen „Störvariablen“ des sozialen Kontextes weitgehend auszuschließen.

Dieses Vorgehen steht im Widerspruch zu Wilhelm Wundts Einsicht, dass psychologische Phänomene nicht alleine durch naturwissenschaftliche Methoden und rein experimentelle Laborstudien zu erklären sind. Wundt, der Begründer der experimentellen Psychologie, hatte daher mit seiner 10-bändigen Völkerpsychologie eine kultur- und sozialwissenschaftliche Ausrichtung der Psychologie gefordert (Wundt, 1900-1920). Diese ist jedoch über Jahrzehnte hinweg auf wenig Zustimmung bei den inzwischen zunehmend naturwissenschaftlich orientierten Psychologen gestoßen (vgl. Graumann, 2006; Komadt, in diesem Band).

Da man die Bedeutung des Kontextes für individuelles Handeln (zumindest aufgrund von Alltagswissen) kennt, wird in der experimentellen Psychologie versucht, (vermeintlich relevante) Kontexteinflüsse zu kontrollieren, um zu universell gïltigen Aussagen zu gelangen. Hier besteht ein Widerspruch zwischen den Zielen und den Möglichkeiten experimenteller psychologischer Laborforschung. Trotz systematischer Kontrolle der Kontextvariablen können die Befunde nicht generalisiert werden. Wenn psychologische Untersuchungen nur innerhalb eines Kulturkontextes und damit verbundenen kulturspezifischen (und historischen) Situationen und Deutungssystemen erfolgen, sind die Ergebnisse nicht ohne weiteres generalisierbar. In der psychologischen Forschung wurden die empirischen Ergebnisse meistens als universell gültig betachtet, obwohl (häufig geforderte) Replikationen von Experimenten immer noch eine Seltenheit sind. Jedoch werden Probleme der systematischen Kontrolle und damit entstehende Probleme der Generalisierbarkeit psychologischer Befunde nur selten artikuliert.

Viele empirische Befunde der Psychologie haben zudem einen kulturspezifisch westlichen „Bias“ (d.h. systematischen Fehler). Dies beruht u.a. auf den theoretischen Ansätzen, den Methoden und den untersuchten Stichproben. Beispielsweise sind an den meisten Studien europäisch-amerikanische College Studenten der weißen Mittelschicht als Untersuchungsteilnehmer beteiligt. Die Mehrheit der Weltbevölkerung lebt jedoch außerhalb der USA und Europas und ist durch ganz andere ökonomische, soziale und kulturelle Bedingungen geprägt. $\mathrm{Ob}$ daher andere Untersuchungsmethoden anzuwenden und die Ergebnisse anders $\mathrm{zu}$ interpretieren sind, ließe sich vielleicht durch entsprechende Studien klären. Damit verbunden ist aber die Frage, in wieweit sich vorgeblich „kontextfreie" experimentelle Befunde überhaupt auf Personengruppen aus anderen Kulturen generalisieren lassen. Dies ist noch weitgehend unbekannt, und wird erst in den letzten Jahren überhaupt als eine relevante Fragestellung gesehen.

Ein bisher weitgehend außerhalb der psychologischen mainstream-Forschung erfolgender Versuch, den Kontext systematisch mit einzubeziehen, wird von der kulturvergleichenden Psychologie vertreten (vgl. Poortinga, 2007; Berry, Poortinga, \& Pandey, 1997; Rothbaum \& Wang, in diesem Band; Grossmann \& Grossmann, in diesem Band; Kornadt, in diesem Band; Schwarz, in diesem Band; Friedlmeier, in diesem Band; Eckensberger, in diesem Band). Dadurch sind einige psychologische Theorien inzwischen durch Kulturvergleiche (u.a. mit afrikanischen, asiatischen, und anderen nichtwestlichen Kulturen) teilweise modifiziert worden. Das gilt für die Forschung zu Kognition und Sprache (Song, Lüer, \& Lass, 2007; Helfrich, 2007), zum Lemen (Hesse, 2007), zur kognitiven Entwicklung (Dasen, 2007; Mishra \& Dasen, 2005), sowie zur motivationalen (Komadt, 2007), emotionalen (Friedlmeier \& Matsumoto, 2007; Trommsdorff, 2006a, Trommsdorff $\&$ Cole, in Druck), der moralischen (Keller \& Krettenauer, 2007) und der sozialen (Leyendecker \& Schölmerich, 2007; Trommsdorff, 2007a) Entwicklung. Inzwischen liegen zahlreiche eindrucksvolle empirische Befunde zu Universalien und zu Kulturunterschieden u.a. in Wahmehmung, Kognition, Gedächtnis, Lemen, Motivation, Emotion, sozialem Handeln und Selbstkonzept vor (vgl. Übersicht in der dreibändigen Enzyklopädie zur kulturvergleichenden Psychologie von Trommsdorff \& Kornadt, 2007a, 2007b, 2007c). Dabei kommen experimentelle Untersuchungen keineswegs zu kurz.

Im Labor kann die psychologische Bedeutung verschiedener sozio-kultureller Kontexte in ihrer Funktion für psychologische Prozesse teilweise systematisch geprüft werden, wenn von vormherein Kulturvergleiche als Methode für die Datenerhebung erfolgen. Zum Beispiel lässt sich die Wirkung des kulturellen Kontextes auf die subjektive Wahmehmung, Urteilsbildung oder auf interpersonales Verhalten experimentell im Kulturvergleich der Aufmerksamkeitsfokussierung asiatischer und amerikanischer Untersuchungsteilnehmer prüfen. Während Asiaten eher den Kontext beschreiben, lenken amerikanische Probanden ihre Aufmerksamkeit eher auf agierende Objekte (Nisbett, 2003). In asiatischen Kulturen kommt vor allem dem sozialen Kontext, in dem der Einzelne handelt, eine größere Bedeutung zu. Der Einzelne erlebt sich dort nicht wie in westlichen Kulturen als unabhängig (independent) von anderen Personen und vom sozialen Kontext sondern als eingebunden (interdependent). Entsprechend sind je nach kulturellem Kontext unterschiedliche Werte der Autonomie und Verbundenheit vorherrschend und handlungswirksam (Rothbaum \& Trommsdorff, 2007; Trommsdorff \& Rothbaum, 2008; Rothbaum \& Wang, in diesem Band) 
Systematische Kulturvergleiche erlauben u.a. einen möglichen westlichen Bias in den Theorien aufzudecken und konfundierte Variablen zu entkonfundieren. Dazu ist die systematische Verwendung kulturvergleichender Methoden erforderlich, auch, um die funktionale Äquivalenz der Erhebungsmethoden und der Verfahren zu sichem. Voraussetzung dafür sind aufwändige Vorarbeiten, u.a. Überseizungen und Rückübersetzungen und diverse Äquivalenzprüfungen (z.B. kulturübergreifende Konstruktäquivalenz) (vgl. Trommsdorff \& Mayer, 2005; Van de Vijver, 2007; Van de Vijver \& Leung, 1997; Eckensberger, 2008)

Um den erheblichen methodischen und organisatorischen Aufwand kulturvergleichender Feldforschung möglichst $\mathrm{zu}$ vermeiden, verzichtet man neuerdings in einigen Untersuchungen von vornherein auf den ,natürlichen“ kulturellen Kontext, bleibt bei den weißen Mittelschichtstudenten und versucht, durch ,priming " Experimente bestimmte kulturspezifische Repräsentationen, die theoretisch als besonders bedeutsam angenommen werden, möglichst kontrolliert wirksam zu machen. Zum Beispiel wird die Salienz einer kulturspezifischen (independenten versus interdependenten) Selbstauffassung durch ,priming“ in einigen experimentellen Studien aktiviert. Damit sollen Zusammenhänge mit bestimmten kognitiven, emotionalen und motivationalen Prozessen geprüft werden, wie sie sich in der kulturvergleichenden Psychologie als relevant erwiesen haben (z.B. Markus \& Kitayama, 1991) (zur kritischen Analyse von Studien zum Individualismus und Kollektivismus und zur Independenz und Interdependenz von Selbstauffassungen vgl. Oysernan, Coon, \& Kemmelmeier, 2002). Einige empirische Befunde sprechen dafür, dass durch „priming“ bestimmte Repräsentationen aktiviert werden können (vgl. Metaanalyse von Oyserman \& Lee, 2008). Allerdings ist ungeklärt, ob und welche Repräsentationen bei Angehörigen verschiedener Kulturen besonders gut oder nur unzureichend ,geprimt“ werden können. Vor allem ist höchst zweifelhaft, ob mit dieser Methode der kulturelle Kontext der Probanden, und vor allem deren lebenslange Erfahrung in diesem spezifischen Kontext, ersetzt werden können, ohne weitere historische, sozio-kulturelle und ökonomische Kontextbedingungen zu berücksichtigen. Es bleibt also der Sachverhalt, dass das „priming" im Gesamtkontext der jeweiligen Kultur erfolgt.

Unklar ist auch, welche (generalisierbaren) psychologischen Prozesse bei Konstanthalten von Umweltbedingungen im Labor auftreten und ob diese (mit denen unter natürlichen kulturellen Bedingungen auftretenden Prozessen) noch funktional äquivalent sind. Wenn sozio-kulturelle und historische Bedingungen außerhalb des Labors andere psychologische Phänomene bewirken als andemfalls unter isolierten Laborbedingungen entstehen, wäre zu prüfen, worin die Unterschiede bestehen und auf welche Prozesse diese zurückzuführen sind, und zwar unter Berücksichtigung sowohl psychologischer als auch sozialwissenschaftlicher Theorien. Die Frage ist hier also, ob im Labor ein ,Mehrwert" des natürlichen Kontextes ignoriert wird. Bei der Prüfung dieser Frage sind bestimm- te methodologische Anforderungen an makro- und mikrosoziologische sowie auch an psychologische Analysen zu stellen. Es ist zunächst davon auszugehen, dass Daten von Individuen nicht unabhängig von dem Kontext der Datenerhebung (im Labor, der Erhebungssituation im Feld etc.) und nicht unabhängig von dem individuell erfahrungsbedingten Kontext sind.

Generell besteht hier das Problem verschiedener Analyseebenen in der Psychologie und Soziologie. Strikt individualistische (reduktionistische) Ansätze übersehen die Besonderheiten von Phänomenen und Dynamiken der Makroebene. Auf der anderen Seite übersehen rein sozio-strukturelle Ansätze die Besonderheiten auf der Ebene individueller Akteure. Kulturvergleichende Methoden der Psychologie können jedoch eine Brücke zwischen beiden Ebenen sein.

Zusammenfassend lässt sich sagen, dass eine kontextfreie Psychologie kaum einen weiterfihrenden Beitrag zur sozialwissenschaftlichen Forschung erbringen kann. Im Folgenden soll zunächst an Beispielen diskutiert werden, ob hingegen eine psychologische Forschung, die von psychologischen Prozessen bei den individuellen Akteuren ausgeht und die die Bedeutung des sozio-kulturellen Kontextes mit berücksichtigt, einen (gewissen) Beitrag zur Aufklärung sozialer Phänomene leisten kann. Dabei werden zuerst soziale Systeme wie Institutionen und des Weiteren sozio-ökonomische Phänomene wie sozialer Wandel und demographische Veränderungen behandelt.

\subsection{Beitrag der kontextbezogenen kulturinformierten Psychologie zur} Erforschung sozialer Systeme

Welchen Beitrag leistet eine kontextbezogene Psychologie für die Untersuchung sozialer Systeme? Um von vornherein ein Missverständnis auszuräumen: Die Untersuchung von sozialen Systemen wie z.B. Institutionen kann nicht Gegenstand der Psychologie sein („Das Ganze ist mehr als die Summe seiner Teile“). Wie oben gesagt, ist die Analyse von Institutionen (Rechts- oder Wirtschaftssystem auf der Makroebene) Untersuchungsgegenstand der Soziologie.

Solche Analysen zu komplexen sozialen Einheiten (also auch zu Gruppen, Klassen, Vereinen) können jedoch teilweise von psychologischer Forschung profitieren. So können psychologische Modelle als Heuristik für Analysen auf der Makroebene (unter Verwendung soziologischer Konzepte) brauchbar sein. Auch können aus psychologischer Sicht Bedingungen für Beziehungen zwischen individuellen Akteuren und Institutionen ein interessantes Forschungsfeld sein. Beispielsweise lässt sich untersuchen, aufgrund welcher psychologischen Bedingungen der Einzelne in der Institution wie und mit welchen Ergebnissen (für sich selbst und für die Institution) handelt. 
So wäre eine theoretisch relevante Bedingung, ob und inwieweit die individuellen und kollektiven Überzeugungen und Ziele übereinstimmen und eine Identifikation mit der Institution erfolgt (Trommsdorff, 1995a). In diesem Zusammenhang ist eine Untersuchungsfrage, wie sich dies auf die politische Partizipation, das Wahlverhalten und Anpassungen an sozialen Wandel auswirkt. Dies ist z.B. im Fall von Akkulturationsprozessen bei Migranten oder von Transformationsprozessen nach der politischen Wende von 1989 relevant. Eine andere
Frage

ven Zielen die Bereıtschaft des Einzelnen variiert, aktiv zur Förderung der Institution beizutragen (durch eigene Investitionen, ehrenamtliche Tätigkeiten etc.), oder primär eigene Interessen zu verfolgen auch unter Inkaufnahme oder gar der
Intenti

sich die Frage nach der Entstehung und Legitimationsbasis von Institutionen als Bedingung für deren Funktionieren sowie die Frage nach dem Wandel von Institutionen.

Die auf soziale Institutionen und deren Funktion bezogenen subjektiven Bewertungen und Attribuierungen sind u.a. von individuellen Bedingungen (Persönlichkeitsmerkmalen, Werten, Einstellungen) sowie auch von sozio-kulturellen Faktoren (Wirtschaftserfolg, Transparenz des politischen Systems) abhängig und beeinflussen indirekt (vermittelt über die subjektive Akzeptanz) die relative Stabilität der jeweiligen Institution. Institutionen sind ihrerseits jedoch auch immer Teil größerer Systeme und daher nicht isoliert wirksam. Eine Frage ist somit, ob z.B. die Akzeptanz der Autonomie internationaler Behörden (und damit der potentiellen Einschränkung eigener nationaler Autonomie) (z.B. bei Finanz- oder Umweltfragen) im internationalen Vergleich verschieden ist und von kulturellen Werten (wie Individualismus, Autonomieorientierung) mit beeinflusst wird (Röhl, Trommsdorff, Vainre, \& Heikamp, 2008). Das sind psychologische Fragen, die sich zur Untersuchung der Funktion sozialer Systeme eignen. Die Akzeptanz von Institutionen hat Folgen für die individuelle Zielbindung (commitment) und darauf bezogenes Verhalten sowie auch für die Bereitschaft, dysfunktionale Komponenten von Institutionen zu erkennen und einer „,Reparatur" zu unterziehen. Solche Reparaturvorhaben können durchaus von psychologischen Modellen der Problemwiederum die Analyseebenen profitieren (Trommsdorff, 2000). Dabei müssen jedoch wiederum die Analyseebenen unterschieden sowie kulturelle Faktoren (wie NorÄhnlich inüsten Werthaltungen) berücksichtigt werden.

Ahnlich Inüssten Untersuchungen zur Funktion von Bildungssystemen vermittelnde individuelle Prozesse der Akzeptanz, Zielbindung und Motivation, Modellwirkung oder ser soziale Prozesse, z.B. der Kommunikationsformen, der berücksichtigen. Aktuelle Vozialen Unterstützung in informellen Gruppen mit berücksichtigen. Aktuelle Vorhaben zur Verbesserung des Bildungssystems beziehen sich zwar vorwiegend auf die Funktion von Institutionen (wie Schulund Hochschulsystem) für den Lern- und Leistungserfolg des Einzelnen. Solche Fragen lassen sich jedoch nicht ohne eine Berücksichtigung von vermittelnden sozialen Prozessen der Familie, Schulklasse, Schule, Region sowie von vermittelnden psychologischen Prozessen bei den individuellen Akteuren (Lehrer, Eltern, Schüler) bearbeiten (Engeser, Rheinberg \& Möller, 2009). Erst dann können solche Fragen besser behandelt werden wie: Warum zeigen die deutschen PISA-Ergebnisse (PISA-Konsortium, 2008) ein deutliches Nord-Süd-

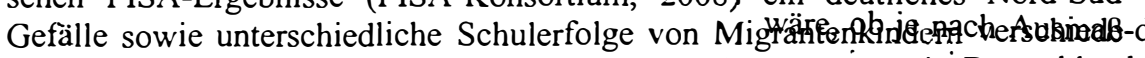
ner ethnischer Herkunft? Warum haben vietnamesische Schüler in Deutschland einen höheren und türkische Schüler einen geringeren Schulerfolg (Statistisches Bundesamt, 2008)? Warum zeigen koreanische und japanische Kinder höhere

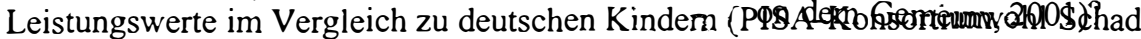

Hier sind u.a. unterschiedliche Einstellungen der Eltern zur Institution der Schule, ein unterschiedlicher Status der Rolle des Lehrers und unterschiedliche Leistungswerte wirksam. Dies lässt sich unter Einbeziehung kollektiver, tradierter kultureller Werthaltungen wie familiale Verpflichtungen und unter Berücksichtigung eines nichtwestlichen Konzeptes einer auf den Erfolg der Familie (und nicht des einzelnen) gerichteten Leistungsmotivation erklären (Hesse, 2007; Helmke, 2008; Komadt, 2007; Trommsdorff, in Druck). Eine nur eurozentrisch orientierte Leistungsmotivationstheorie, die nur den individuellen Erfolg fokussiert, scheitert hier.

Bei weiter gehenden Fragen zur Institutionenbildung (z.B. im Fall einer grundlegenden Neuordnung wie im Transformationsprozess nach der Wende, vgl. Lepsius, 1996) und zur Legitimationsgrundlage von Institutionen geht es auch (aber nicht nur) um psychologische Phänomene wie das Ausmaß von subjektiver Akzeptanz oder Ablehnung von Institutionen (vgl. die oben skizzierten Folgen einer Diskrepanz zwischen individuellen und kollektiven Zielen), um Attribuierung von Autonomie und Verantwortung, um wahrgenommene Zuverlässigkeit der Sanktionierung von Normen (als Grundlage für Vertrauen) oder um den erwarteten Nutzen z.B. bei freiwilliger Zugehörigkeit zu einer Institution (z.B. zu Parteien oder Kirchen) (Trommsdorff, 2000).

Eine interessante, bisher unzureichend beachtete Überlappung psychologischer und sozialwissenschaftlicher Forschung erweist sich in Untersuchungen zur individuellen und kollektiven Intentionalität (Absichtlichkeit). Individuelle Ziele können unterschiedlich stark mit den kollektiven Zielen übereinstimmen, sie können wie im Fall von sozialen Dilemmata (z.B. „Gemeingutprobleme"; Paniksituation) miteinander konfligieren, sie können sich gegenseitig beeinflussen (verstärken oder abschwächen), sie werden von verschiedenen Faktoren (innerhalb der Person, des Kollektivs bzw. der Gruppe und der situativen Bedıngungen) beeinflusst und sie beeinflussen individuelles und kollektives Verhalten. 
Ausgangspunkt psychologischer Ansätze zur kollektiven Intentionalität (wie sie sich z.B. in kulturellen Werthaltungen erkennen lassen) sind die individuellen Akteure und ihre jeweiligen Ziele, Einstellungen und Verhalten auch gegenüber sozialen Institutionen. Dabei haben die Institutionen die Funktion, die Aktivitäten der jeweiligen Individuen zu bündeln und deren Verhaltensspielraum durch Sanktionen einzuschränken, um koordiniertes Handeln im Gesamtinteresse zu ermöglichen. Aus handlungstheoretischer Sicht lassen sich dann Bedingungen für Diskrepanzen von individuellen und kollektiven Zielen präzisieren und Vorhersagen für individuelles Verhalten ableiten (Trommsdorff, 2007b). Für die Untersuchung von kollektiver Intentionalität in formalen Gruppen oder Institutionen ist hingegen erforderlich, die entsprechenden Phänomene (z.B. Kontrollund Sanktionsmechanismen) unter Verwendung von soziologischen Analyseeinheiten und Ansätzen zu erklären.

Zusammenfassend lässt sich sagen, dass eine kulturinformierte Psychologie durchaus einen Beitrag zu den Sozialwissenschaften und sogar zur Institutionenforschung leisten kann. Allerdings sind die Grenzen der Psychologie bzw. ihrer Brauchbarkeit für die Sozialwissenschaften erreicht, wenn es global um die Untersuchung von sozialen Systemen (wie Kollektiven und Institutionen) geht.

\subsection{Sozio-ökonomischer Wandel als Forschungsgegenstand der} kulturinformierten Psychologie

Während die Psychologie nur in Grenzen und indirekt einen substantiellen Beitrag zu Analysen von Institutionen leisten kann, fordern andere soziale Phänomene psychologische Analysen geradezu heraus, wie z.B. Prozesse und Folgen sozio-ökonomischen und politischen Wandels. Wandel kann auf allen Ebenen (Individual-, Gruppen-, Institutionen-, Länderebene) erfolgen, er kann individuell oder kollektiv intendiert sein, er kann durch individuelles und kollektives Handeln entstehen und auf verschiedenen miteinander verbundenen Ebenen verstärkt oder verhindert werden. Sozialer Wandel kann abrupt erfolgen und tiefgreifende Veränderungen im sozialen Kontext und den Lebensbedingungen des Einzelnen bedeuten. Sozialer Wandel kann jedoch auch allmählich und graduell erfolgen. Sozialer Wandel kann Einschränkungen und Risiken aber auch Chancen für den einzelnen implizieren. Dies kann u.a. vom Lebensalter und verfügbaren Ressourcen (in der Familie und Beruf) abhängen. Entsprechend werden die Anforderungen und die Anpassungen an sozialen Wandel individuell unterschiedlich wahrgenommen. Daher sind psychologische Analysen erforderlich, die beides mit einbeziehen, das Individuum und die Gesellschaft.

In diesem Sinne hat Glen Elder (1998) mit seinen Untersuchungen zu $\mathrm{Zu}$ sammenhängen zxischen sozio-ökonomischem Wandel und individueller Ent- wicklung über die Lebenss panne schon relativ früh Grundlagen eines tragfähigen theoretischen und methodischen Ansatzes zum sozialen Wandel aus sozialwissenschaftlicher und psychologischer Sicht geschaffen. Dabei geht er von Veränderungen in der Entwicklung des Einzelnen über die Lebensspanne aus. Seine einflussreiche Studie "The Children of the Great Depression" (1974) belegt die Bedeutung von prospektiven Längsschnittstudien mit Fokus auf sozio-ökonomischen Veränderungen und deren Auswirkungen auf den individuellen Lebens verlauf. Auch seine folgenden Studien zu Strukturveränderungen der Landwirtschaft in den USA mit Konsequenzen für den Arbeitsmarkt, Familienstruktur und für psychologische Belastungen des Einzelnen zeigen, dass durch Einbeziehung psychologischer Theorien zu familialen Sozialisationsbedingungen und den dort vermittelten Ressourcen die individuelle Stressverarbeitung erklärt werden kann. Dazu sind jedoch komplexe theoretische Modelle zum Verhältnis von sozialem Wandel und individueller Anpassung zugrunde zu legen (vgl. Elder, 1998).

Ein grundlegender sozialer, ökonomischer und kultureller Wandel erfolgte durch die plötzlichen und unenwarteten Transformationsprozesse mit dem Zusammenbruch der ehemaligen DDR in den neuen Ländern. Dieser Wandel stellte als ein Systemumbruch einen Sonderfall dar, der über einen "nur" sozioökonomischen und politischen Wandel weit hinausging und neue Fragen für die Sozialwissenschaften und die Psychologie aufgeworfen hat. Daher wäre hier eine enge Zusammenarbeit zwischen Psychologen und Sozialwissenschaftlem erforderlich gewesen. Beide Disziplinen waren darauf aber zunächst nicht vorbereitet. Die Transformation der ehemaligen DDR als ein Sonderfall tiefgreifenden sozioökonomischen und politischen Wandels wurde zunächst in seiner Bedeutung für die sozialwissenschaftliche und psychologische Forschung nicht erkannt.

Was ist erfolgt? Auf Anregung des Wissenschaftsrates (1990) wurde im Jahre 1991 die „Kommission für die Erforschung des sozialen und politischen Wandels in den neuen Bundesländern e.V. (KSPW)" als gemeinnütziger Verein mit 13 Vorstandsmitgliedern verschiedener Disziplinen aus Ost- und Westdeutschland gegründet. Die Finanzierung erfolgte für fünf Jahre. Der Vorstand vergab und veröffentlichte Forschungsarbeiten und stellte selbst Berichte über die Transformation zusammen. Neben Monographien und Sammelwerken zu speziellen Themen (36 Bände) entstanden anfangs 150 Forschungsberichte (zu kleineren Projekten) und danach 103 größere Projekte. Die vom Vorstand herausgegebenen Abschlussberichte der KSPW (Bertram, Nickel, Niedermayer, \& Trommsdorff, 2006) umfassen sechs Bände mit übergreifenden Themen: „ArTrommsdorff, 2006) umfassen “ce ,Ungleichheit und Sozialpolitik“, „Politisches System" "Individuelle Entwicklung, Bildung und Berufsverläufe“, „Städte und Regionen", „Umwandlung der Arbeits- und Sozialordnung“. Explizit und primär psychologische Analysen wurden nur in einem der sechs Berichte vorgelegt (Hormuth, Heinz, Komadt, Sydow, \& Trommsdorff, 1996) und Beiträge zu psy- 
chologischen Prozessen erfolgten in den Berichten nur vereinzelt. Einen umfassenden Überblick über die Arbeiten der KSPW sowie eine kritische Analyse der zugrunde liegenden Gesellschaftskonzeptionen (bzw. Modernisierungsthesen) im Zusammenhang mit einer Theorie- und Forschungsgeschichte der Soziologie gibt Uta Gerhardt (2009).

Die vorwiegend allgemeinpsychologisch orientierten deutschen Psychologen haben sich kaum mit der Wende und deren Wirkung auf die ost- und westdeutsche Bevölkerung befasst. Sie haben die einmalige Gelegenheit, die psychologischen Folgen tiefgreifenden sozio-politischen, ökonomischen und damit auch kulturellen Wandels mit seinen veränderten Ziel- und Wertorientierungen zu untersuchen, nur begrenzt genutzt. So liegt kaum psychologische Forschung vor zu subjektiv erlebten Veränderungen und deren Bewertung, dem damit verbundenen Kontrollerleben und Wohlbefinden, den Bedingungen politischer Partizipation. Grundlegende Veränderungen in Erziehungs- und Bildungssystem und in der Familienstruktur hatten psychologische Folgen für die Sozialisation in der Schule und Familie und sind relevant für verschiedene soziologische und politikwissenschaftliche Fragen der Wirkungen des Transfornationsprozesses, z.B. auf soziale Ungleichheit; Arbeitsmarkt; demographische Entwicklung. Einige dieser Fragen wurden schließlich auf Grundlage psychologischer Ansätze von Identitätsentwicklung, Stressbewältigung, Akkulturation, Gerechtigkeitserleben, Zukunfts- und Kontrollorientierung, Entscheidungsforschung, Risikobereitschaft, sozialer und kognitiver Kompetenz, Konfliktentstehung und -regelung in Interund Intragruppenprozessen bearbeitet (Trommsdorff, 1994; 1995a; Trommsdorff \& Chakkarath, 1996).

Die Chance fuir Psychologen, ein quasi-experimentelles Design einer Feldstudie zu nutzen, wäre fast völlig vertan worden, hätte nicht die Deutsche Forschungsgemeinschaft ein Schwerpunktprogramm (,Kindheit und Jugend vor und nach der Wende - Wissenschaftler untersuchen Sozialisation in Ost und West") aufgelegt, das Psychologen aus Ost- und Westdeutschland die Gelegenheit gegeben hat, im Längsschnitt wichtige Fragen der psychologischen Folgen der Transformation zu untersuchen.

In diesen Studien zeigte sich u.a., dass die „Wende“ Wirkungen hatte, die sich im Zeitverlauf für verschiedene Kohorten wiederum änderten. Die Elterngeneration der neuen Länder war anfangs zunehmend verunsichert; bei ihren Kindern und Jugendlichen zeigte sich im Vergleich zu westdeutschen Altersgruppen zunächst eine geringere Ausprägung positiven Sozialverhaltens (Trommsdorff \& Komadt, 1995). Nach einigen Jahren verschwanden diese Unterschiede jedoch und das Sozialverhalten west- und ostdeutscher Kinder und Jugendlicher glich sich an (Trommsdorff, Komadt, \& Hessel-Scherf, 1998). Es kann angenommen werden, dass die in den neuen im Vergleich zu den alten Bundesländern stärker ausgepràgte Familienorientierung und die erlebte mütterliche Wärme als Res- source über die Wende hinaus als Schutzfaktor für Kinder und Jugendliche im Transfonmationsprozess wirksam war.

Neuere Studien von Silbereisen, Pinquart und Tomasik (in Druck) zu den Veränderungen nach der Vereinigung in Ost- und Westdeutschland (PostTransformations-Periode) knüpfen wieder an dem Ansatz von Glen Elder an. Die Autoren untersuchen gemäß dem Modell der individuellen Anpassung an sozialen Wandel (Globalisierung, Individualisierung, Pluralisierung von Lebensformen u.a.) Zusammenhänge zwischen Anforderungen und psychosozialer Entwicklung, die sich einerseits in den Anpassungsprozessen und andererseits in den verfügbaren Ressourcen zeigen.

Sozio-ökonomischer und politischer Wandel eines ganzen Systems kann sich, vermittelt über verschiedene Institutionen und infonnelle soziale Netze, auf die Lebens- und Entwicklungsbedingungen des Einzelnen auswirken. Verändern sich damit aber auch individuelle Werthaltungen, Einstellungen und Verhaltenspräferenzen? In seinen inzwischen mehr als eine Dekade umfassenden Längsschnittstudien haben Chen und Chen (in Druck) nachgewiesen, dass im Zusammenhang mit dem dramatischen sozio-ökonomischen und politischen Wandel in der VR China zum Beispiel traditionelle Werte und Verhaltensweisen wie Bescheidenheit und Zurückhaltung (shyness) zur Aufrechterhaltung von Harmonie in der Gruppe gegenüber „modernen“ Werten der Durchsetzungsfähigkeit weniger wichtig geworden sind. Damit haben sich die Entwicklungsbedingungen und folgen für Kinder und die nachwachsende Generation entsprechend verändert. Als sozial kompetent eingestufte Kinder zeigen heute, anders als vor mehr als einer Dekade, geringere shyness. Je nach Anforderungen durch den sozialen Wandel können bestimmte Verhaltensweisen (Zurückhaltung) ihre erwünschten Funktionen verlieren und andere (ja sogar entgegengesetzte) Verhaltensweisen werden wichtiger und erwünscht. Die Längsschnittstudien von Chen und Mitarbeitern belegen die durchgreifende Wirkung des sozio-ökonomischen Wandels (auf der Makroebene), die vermittelt über den Wertewandel und veränderte Sozialisationsbedingungen die individuelle sozio-emotionale Entwicklung beeinflussen. Regionale Unterschiede im sozialen Wandel und deren Wirkung auf die individuelle Entwicklung belegen zudem den hohen Einfluss des sozio-kulturellen Kontextes.

Fragen zur gegenwärtigen Wirtschaftskrise gehen ebenfalls über Analysen eines „nur“ allmählichen, stetigen sozio-ökonomischen Wandels hinaus, weil vorgeblich unvorhersehbare dramatische Umbrüche erfolgt sind, die global die gesamte Weltwirtschaft und damit die Lebensbedingungen von Einzelnen über Jahre hinweg beeinträchtigen. Um die Entstehung der Krise zu erklären, verwenden die Sozial- und Wirtschaftswissenschaften häufig psychologisierende Konzepte wie Gier, Optimismus und Risikofreude. Einen Ausweg aus der Krise sehen sie in der Kooperationsbereitschaft und im Vertrauen in die Funktionsfä- 
higkeit der Institutionen, also in der oben skizzierten Legitimationsgrundlage funktionierender Systeme. Allerdings ist deutlich geworden, dass das Vertrauen durch übergeordnete Institutionen geschützt werden muss, um den Egoismus von nicht kooperierenden "Trittbrettfahrern", die das Gemeingut schädigen, zu kontrollieren und gleichzeitig die intrinsische Motivation der kooperierenden Akteure zu erhalten. Hier wird wieder der Grundkonflikt von Gemeingutproblemen (als Typ sozialer Dilemmata) angesprochen, der sich hier allerdings weniger in informellen persönlichen als vielmehr in anonymitätsbasierten Interaktionen vollzieht und damit eine andere Dimension erreicht.

Die Lösung von ökonomischen Problemen ist immer auch mit psychologischen Prozessen verbunden. Empirische psychologische Befunde aus neuerer kulturvergleichender Werte-Forschung zu Bedingungen und Folgen egoistischmaterialistischer im Vergleich zu kooperativ-materialistischen Orientierungen belegen, dass kulturell und individuell vorherrschende Werthaltungen die Art der Lösung ökonomischer Probleme beeinflussen. In Ländern mit wettbewerbsorientiertem Kapitalismus (USA, England, Australien, Kanada) im Vergleich zu Ländern mit eher kooperativem Kapitalismus (Deutschland, Österreich, Norwegen) erfolgt die Lösung ökonomischer Probleme wie Arbeitslosigkeit weniger kooperativ (Schwartz, 2007). Andere psychologische Studien (zum US amerikanischen korporativen Kapitalismus) belegen, dass materielles Gewinnstreben und soziale Orientierung (wie Prosozialität) kaum miteinander zusammenhängen, weil sie vermutlich auf unterschiedlichen Motivsystemen beruhen (Kasser, Cohn, Kanner, \& Ryan, 2007). Diese Befunde weisen darauf hin, dass kooperationsfördernde Institutionen bei einer solchen Konstellation wichtige Steuerungsfunktionen haben. Wenn hingegen eine egoistische Trittbrettfahrermentalität zu Lasten der kooperierenden Mitglieder geduldet und verstärkt wird, kann das System zusammenbrechen. Das entspricht der Annahme, dass soziale Institutionen und Strukturen zusammen mit den kulturellen und individuellen Werthaltungen und der Motivation des Einzelnen die Richtung ökonomischen Handelns beeinflussen.

Zusammenfassend lässt sich anhand dieser Beispiele der signifikante Beitrag psychologischer und kulturinformierter Forschung (zur Persönlichkeitsentwicklung oder zu Zusammenhängen zwischen Werten und Verhalten) zu sozialwissenschaftlichen Themen der Bedingungen und Folgen sozio-ökonomischen Wandels verdeutlichen.

\subsection{Demographischer Wandel als Problem kulturinformierter Psychologie}

Auch die soziodemografischen Veränderungen bringen wichtige Fragen mit sich, auf die die Psychologie und die Sozialwissenschaften Antworten zu geben hätten. Diese Fragen werden jedoch weniger in der Psychologie als in der Soziolo- gie wahrgenommen. Mit dem deutlichen Rückgang der Geburtenrate in westlichen Industrieländern geht $z$.B. eine zunehmende Lebenserwartung einher. Kein westlicher Wohlfahrtsstaat erreicht heute noch die "Netto-Reproduktionsrate“ von durchschnittlich 2.08 Kindern pro Frau, womit eine stabile nationale Bevölkerung gesichert ist. In diesem Zusammenhang ist auch ein tiefgreifender Wandel der Familienstruktur erfolgt. Familienformen werden zunehmend individualisierter (vgl. Bengtson, 2001; Nave-Herz, 2002; GESIS, 2008; Nauck, 2009, in diesem Band). Einige Beispiele dazu sind: Familien mit drei oder vier Kindern werden zunehmend seltener; Kinder wachsen zunehmend häufig ganz ohne Geschwister, oder mit Kindern des neuen Lebenspartners des geschiedenen Elternteils, oder in Ein-Eltern-Familien auf; die Zahl kinderloser Familien (und Paare) steigt; die Anzahl von Scheidungen und verschiedene Formen partnerschaftlichen Zusammenlebens ohne Ehe nehrmen zu. Eine solche neue Form einer quasiFamilienbildung ist Living Apart Together (LAT). Hier wohnen beide Partner in getrennten Haushalten, sehen sich aber als zusammen lebend. Nach Asendorpf (2008) hat LAT seit 1992 zugenommen; sie nimmt bis zum Ende der weiblichen Reproduktionsphase (40 Jahre) ab und bleibt danach eine eigenständige Lebensform, die allerdings in allen Altersgruppen instabiler als die Ehe und die (ebenfalls gestiegene Häufigkeit von) Kohabitation ist.

Was sind die psychologischen Bedingungen für und die Folgen von solchen Veränderungen der Familienformen aus kulturvergleichender Sicht (vgl. Trommsdorff \& Nauck, 2006)? Wird die zunehmende Individualisierung von Familienformen von einem entsprechenden Wertewandel mit verursacht oder begleitet, der u.a. den Kinderwunsch, die Rolle der Frau und die Vereinbarkeit von Beruf und Familie beeinflusst? Wie ist die abnehmende Geburtenrate auch in asiatischen Ländern trotz dort bestehender hoher Bedeutung von Familienwerten zu erklären? Welche Folgen haben die veränderten Familienformen für die zukünftige Sozialisation und Entwicklung der Kinder und Jugendlichen? Wie wirkt sich die verlängerte Lebenserwartung auf individuelle Anpassungsprozesse der älteren Bevölkerung aus, insbesondere, wenn aufgrund veränderter Familienformen keine Kinder oder Ehepartner verfügbar sind, die Unterstützungsleistungen erbringen könnten? Beschleunigt sich dieser Prozess gegenwärtig? Wird dann eine nichtfamilienbasierte staatlich subventionierte Unterstützung notwendig und welche Folgen hat dies für die individuelle Entwicklung im Alter und für Solidaritätsbeziehungen zwischen den Generationen? Das sind Fragen, auf die sowohl die Psychologie als auch die Sozialwissenschafien Antworten geben müssen.

Generationenbeziehungen sind nicht nur ein soziologisches Phänomen der Beziehung zwischen Kohorten sondern ein psychologisches Phänomen der interpersonalen Beziehungen in familial verbundenen Generationen (Künemund \& Szydlik, 2009). Die Art der Generationenbeziehung hat Implikationen für die 
Transmission von Werten zwischen den Generationen (Albert, 2007; Trommsdorff, 2009a). Psychologische Faktoren der Eltem-Kind-Beziehung (Trommsdorff, 2006b) und kulturelle Werte beeinflussen u.a. die Bereitschaft für die materielle Versorgung der älteren Generation und deren praktische und emotionale Unterstützung (Trommsdorff \& Albert, 2009; Schwarz, Albert, \& Trommsdorff, 2009; Schwarz, in diesem Band). Die Intergenerationenbeziehungen verändem sich in einer demografisch veränderten Gesellschaft und stellen neue Anforderungen an die Gesellschaft und den einzelnen.

Einige der oben genannten Fragen zu den Bedingungen und Folgen des demografischen Wandels werden in dem von der Deutschen Forschungsgemeinschaft (DFG) unterstützten kulturvergleichenden Projekt „Value of Children and Intergenerational Relations (VOC IGR)“ untersucht. Die beiden Principal Investigators (Trommsdorff \& Nauck, 2005) untersuchen mit ihren kooperierenden Teams aus 13 verschiedenen Kulturen im Rahmen einer umfangreichen kulturvergleichenden Mehrgenerationenstudie Bedingungen des demografischen Wandels. Wir haben dabei sowohl psychologische wie soziologische Ansätze in Bezug auf den Kinderwunsch, veränderte Familientormen, Beziehungen zwischen drei familial verbundenen Generationen (Großmutter, Mutter, Jugendliche) verwendet und verschiedene theoretisch abgeleitete Modelle u.a. auch unter Einbeziehung von individuellen Werthaltungen und kontextuellen (u.a. strukturellen) Faktoren geprüft (vgl. Trommsdorff \& Nauck, 2005; Trommsdorff, Kim, \& Nauck, 2005; Trommsdorff, 2009b; Nauck, 2009; Nauck, in diesem Band).

meisten wirtschaftlıch entwickelten Ländem und Regionen heute die sozioökonomische Bedeutung von Kindem keine Rolle mehr spielt (die ökonomischen Kosten von Kindem werden allerdings wahrgenommen) und die emotionale Bedeutung von Kindem deutlich salienter geworden ist (Mayer \& Trommsdorff, 2009). Daher erscheint es plausibel, dass bei dieser veränderten Wertekonstellation nicht (wie traditionell üblich) viele Kinder (bevorzugt Jungen) für die materielle Bedürfnisbefriedigung (z.B. Versorgung im Alter) gewünscht werden, sondem bereits ein oder zwei Kinder (Junge oder Mädchen) eine hohe emotionale Erfüllung bedeuten; zudem haben die Kosten der Erziehung (Zeit, Finanzierung der Ausbildung) und der Wert der Selbständigkeit für Frauen zugenommen, was den Wunsch nach mehr als zwei Kindem begrenzt

Hinsichtlich

gezeigt, dass positıve Eltem-Kınd-Bezıehungen die Weitergabe von elterlichen Werten an die nächste Generation und in westlichen Kulturen auch die Bereitschaft zu elterlicher Unterstützung der Eltem im Alter fördert (Albert 2007; Trommsdorff, 2009a). Im asiatischen Kulturkontext sind jedoch die erlebten Normen und Pflichten der filial piety für die Unterstützungsbereitschaft wirksam; diese gehen über die in westlichen Kulturen üblichen Reziprozitätserwartungen hinaus (Schwarz, Trommsdorff, Albert, \& Mayer, 2005; Schwarz \& Trommsdorff, 2006). Dies weist auf die Bedeutung des Wertes der Familie und der Verbundenheit in asiatischen Kulturen hin. Zwar zeigt sich teilweise auch hier ein Wertewandel; dieser belegt aber keine Transformation und Änderung zu westlichen Lebensbedingungen und Werten.

Zusammenfassend lässt sich zeigen, dass psychologische kulturvergleichende Studien zum demografischen Wandel in Zusammenarbeit mit familiensoziologischer Forschung einen Beitrag zur Aufklärung von Bedingungen und Folgen sozio-ökonomischen Wandels leisten können. Hier wurde deutlich, dass diese komplexen Fragen zum demografischen Wandel, zum Wandel von Familienformen und Generationenbeziehungen sowohl psychologischer als auch soziologischer Ansätze für deren Bearbeitung bedürfen und wie fruchtbar die Zusammenarbeit zwischen beiden Disziplinen sein kann.

\section{Beitrag der Psychologie für Sozial- und Wirtschaftswissenschaften}

Im Folgenden werden einige Beispiele diskutiert, die zeigen, dass psychologische Erkenntnisse und Theorien in sozial- und wirtschaftswissenschaftliche Ansätze eingeflossen sind und wie eine kulturinformierte psychologische Forschung dazu beitragen kann, sozialwissenschaftliche Ergebnisse aufzuklären. Natürlich bestehen auch jeweils Grenzen dieser Ansätze.

Wenn eine Disziplin ein gut etabliertes the hes und methodisches Wissen zur Verfügung hat, ist es nicht einfach, neue Konzepte und Fragestellungen einzuführen. Das gilt z.B. für die Ökonomie mit einem gut abgesicherten Kem wirtschaftswissenschaftlichen Wissens. Dennoch haben sich neuerdings die Sozialwissenschaften und die Ökonomie der Psychologie gegenüber geöffnet und bislang völlig vemachlässigte psychologische Konzepte sowohl in theoretischen Ansätzen als auch in den empirischen Studien eingeführt. Dies wird besonders in der zunehmenden Berücksichtigung psychologischer Variablen (Persönlichkeitsmerkmale wie Big Five; Kontroll- und Zukunftsorientierung) sowie auch psychologischer Fragestellungen (z.B. zu Entwicklungsbedingungen und Veränderungen über die Lebensspanne) im Sozio-oekonomischen Panel (SOEP) deutlich (vgl. Trommsdorff, 2008a; Wagner, Spieß, \& Schupp, 2008: Schupd \& Wagner, in diesem Band).

Als Beispiele für die fruchtbare Berücksichtigung psychologischer Ansätze in den Wirtschaftswissenschaften werden im Folgenden zunächst Studien zum reziproken Altruismus in den Behavioral Economics (Fehr \& Fischbacher, 2005) und dann Studien zur Lebenszufriedenheit im Zusammenhang mit der Glückstoorschung (Frey, 2008) diskutiert. Diesen unterschiedlichen Forschungsrichtungen 
liegt die Erkenntnis zugrunde, dass Emotionen eine bedeutende Rolle im Verhalten zukommt. Im dritten Teil wird daher diskutiert, dass Fragen der Rationalität von Entscheidungen im Lichte neuer psychologischer Ergebnisse (bzw. systematischer „Verzerrungen“") zu sehen sind.

\section{3.] Emotionen und Altruismus}

Rolle der Emotionen und der Empathie in der Psychologie. In der psychologischen Forschung sind Emotionen (abgesehen von frühen motivationstheoretischen Ansätzen) erst nach der kognitiven Wende (auch nachdem klassische austauschtheoretische Ansätze nicht mehr ausreichend erschienen) als wichtige Faktoren für die verschiedensten Verhaltensbereiche (z.B. für prosoziales Handeln, Selbstregulation) erkannt worden. Inzwischen ist auch die Rolle kultureller Kontexte für die Bedeutung verschiedener Emotionen für Verhalten untersucht worden (Friedlmeier, in diesem Band; Friedlmeier \& Matsumoto, 2007; Markus \& Kitayama, 1991; Matsumoto, 2001; Scherer, 1997; Trommsdorff, 2006a; Trommsdorff \& Cole, in Druck).

In der Emotionsforschung wird die Empathie als biologisch fundiertes Fundament menschlichen Zusammenlebens insbesondere des prosozialen Verhaltens gesehen (Bischof-Köhler, 1991; Trommsdorff, 1995b; Eisenberg, 2002; Eisenberg, Fabes, \& Spinrad, 2006). Dies wurde durch die biowissenschaf tliche Himforschung über Spiegelneuronen als Voraussetzung für das Erleben von Empathie und damit als Ressource für zwischenmenschliche Kooperation erhärtet (Rizzolatti \& Sinigaglia, 2008).

Empathie ist die Fähigkeit und Bereitschaft für (aktuelles und antizipiertes) emotionales Miterleben. Dies erleichtert das Verständnis für Bedürfnisse und Ziele des anderen (Theory of Mind; vgl. Bischof-Köhler, 2000, in diesem Band), für Altruismus und kooperative soziale Interaktionen

Durch Empathie miterlebte aktuelle Emotionen des anderen können eigenes Handeln beeinflussen (z.B. Hilf eleistung, Inhibition von Aggression). Auch nur beim anderen antizipierte Emotionen (wie dessen Enttäuschung) können (z.B. vermittelt über Schuldgefuihle) eigenes Verhalten beeinflussen (z.B. Nachgiebigkeit, Regelanpassung). In kulturvergleichenden Studien haben wir gezeigt, dass Empathie eine notwendige (wenn auch nicht hinreichende) Bedingung für prosoziales Verhalten ist (Trommsdorff, Friedlmeier \& Mayer, 2007). Zudem werden bei hoher Empathie aggressive Tendenzen gehemmt (Komadt, 2007). Förderlich für die Entwicklung von Empathie und Altruismus ist eine enge frühe MutterKind-Beziehung (vgl. Eisenberg, 2002; Bischof-Köhler, 1991; Trommsdorff \& Friedlmeier, 1999, in Druck).
Rolle des Altruismus und der Rationalität in der Ökonomie. Inzwischen haben Ökonomen die Rolle von Emotionen für wirtschaftliches Handeln entdeckt und damit einen weiteren Bezug zur Psychologie hergestellt (vgl. Shiller \& Akerlof, 2009). Die Ökonomie hat lange gebraucht, um zu ihren eigenen Wurzeln, der Annahme eines nicht-egoistischen Menschenbildes, zurückzukehren. Adam Smith (1978) hat mit "The Wealth of Nations" lange Zeit in eine Richtung gewirkt: Wettbewerb und wirtschaftlicher Erfolg seien durch das Eigeninteresse der Akteure gekennzeichnet. Diese Annahme wurde Grundlage des Modells des rational handelnden Homo Oeconomicus. So hat die neo-klassische Ökonomie das Eigeninteresse als zentrale Motivation fur wirtschaftliches Handeln betrachtet. Hingegen hatte Adam Smith auch (1969) bereits in „Theory of Moral Sentiments” dargelegt, dass „Sympathy“ ebenfalls eine Emotion ist, die interpersonales Verhalten beeinflusst.

Unter Berücksichtigung der Verhaltenswissenschaften und insbesondere der Psychologie hat die experimentelle Ökonomie psychologische Erkenntnisse auf gegriffen und empirisch nachgewiesen, dass rationales Verhalten keineswegs nur egoistisches Verhalten ist sondern dass rationales Verhalten vielmehr auch die Interessen anderer mit einbeziehen und altruistisch sein kann. Inzwischen werden in dieser neueren verhaltensökonomischen Forschungsrichtung nicht nur experimentelle Labor- sondern auch Feldstudien (unter natürlichen Bedingungen in Schulen, Unternehmen, in traditionellen und modernen Gesellschaften) durchgeführt. Diese erfolgen in Kooperation mit Anthropologen, Sozialpsychologen, Politologen und Soziologen. Dabei werden auch ethnografische und andere Daten einbezogen und Verhaltensbeobachtungen inzwischen auch im Kulturvergleich getestet (vgl. Übersicht bei Gintis, Bowles, Boyd, \& Fehr, 2005).

Die neuere verhaltensökonomische Forschung geht von der These der strong reciprocity aus (Gintis et al., 2005). Die empirischen Befunde zeigen, dass die Kooperation mit anderen dazu führt, dass nicht kooperierende Gruppenmitglieder (also solche, die die anderen Mitglieder und deren Leistungen egoistisch ausnutzen) dafür bestraft werden. „Strong reciprocators are conditional cooperators (who behave altruistically as long as others are doing so as well) and altruistic punishers (who apply sanctions to those who behave unfairly according to the prevalent norms of cooperation)." (Gintis et al., 2005, p.8).

Hier wird also nicht von einem egoistisch oder einem altruistisch handelnden Menschen ausgegangen sondern von einem ,,rational" handelnden, der die Vorteile gemeinsamer Kooperation nutzt. Mit dem Ansatz der ,strong reciproci$t y$ “ wird auch deutlich, was bei der Beurteilung von Verhalten wichtiger ist: faire oder unfaire Ergebnisse oder faire oder unfaire Intentionen der Handelnden. Rationalität wird nach diesem Ansatz eindeutig als ein Verhalten gesehen, das auf Altruismus und Empathie der beteiligten Kooperateure beruht. 
Die Erkenntnis der Bedeutung der psychologischen Faktoren Empathie und Altruismus hat nicht nur die Ökonomie beeinflusst. Auch die Evolutionstheorie hat davon profitiert und diese hat wiederum ökonomische und sozialwissenschaftliche Theorien beeinflusst. Ansätze zur genetisch-kulturellen Koevolution behaupten die Überlegenheit von kooperativen gegenüber nicht-kooperativen Gesellschaften. Forschung zur kulturellen Selektion von Gruppen zeigt, dass Kulturen, die Reziprozität fördern und weitergeben, solchen Kulturen überlegen sind, die dies nicht tun (Gintis et al., 2005). Die These der inklusiven Fitness, eine Grundlage biologischer Modelle menschlichen Verhaltens (Dawkins, 1976) und die These vom "selfish gene“ gelten inzwischen als überholt. Auch nicht Verwandte können einander helfen und einander Opfer bringen.

Auch entwicklungspsychologische Studien haben gezeigt, dass Faimess positive Emotionen mehr beeinflusst als der tatsächliche materielle Gewinn (Arsenio, 2008). Dies steht im Widerspruch zur klassischen Spieltheorie, die eine Präferenz des eigenen materiellen Nutzens (u. U. auch zu Lasten von Faimess) annimmt; gleichzeitig spricht dies für einen hedonistischen Wert der Faimess als „rationaler" Grundlage für Verhalten.

Diese neue Sichtweise auf eine ,eingeschränkte Rationalität“ als Grundlage von sozialem und ökonomischem Verhalten wertet die Rolle von Emotionen signifikant auf und unterstreicht die Bedeutung psychologischer Konzepte der Empathie und des Altruismus. Diese könnten für die verhaltensökonomische Forschung in Zukunft weiter fruchtbar werden.

\subsection{Lebenszufriedenheit als Untersuchungsgegenstand der kulturvergleichenden Psychologie und der Sozial-und Wirtschaftswissenschaften}

Schon zu Beginn der Untersuchungen des Sozio-oekonomischen Panel (SOEP) (vgl. Schupp \& Wagner, in diesem Band) sind einige wenige psychologische Indikatoren neben rein ökonomischen und sozialwissenschaftlichen Indikatoren in die Methoden und Datenerhebung eingegangen. Auch die Lebenszuf riedenheit gehörte dazu. Damit sollte das individuelle Wohlbefinden erfasst werden, was häufig in Zusammenhang mit sozialer Ungleichheit und materiellem Wohlstand untersucht wurde. Inzwischen ist die Forschung zur Lebenszufriedenheit eng verbunden mit der „Glücks"-Forschung (,happiness“) (vgl. „positive psychology"). Die Psychologie hat differenzierte theoretisch fundierte Messverfahren bereitgestellt, um Lebenszufriedenheit zu operationalisieren. Das Konzept ist fiir ökonomische, politische und soziale Fragen angewendet worden, wobei die theoretischen Ansätze der Psychologie zur Erklärung von Glück und Zufriedenheit allerdings bisher nur teilweise berücksichtigt wurden.
Was ist die Grundlage von Glück? Die Varianz für die Definitionen von Glück ist erheblich. Politiker verfolgen das Ziel, ihre Wähler zufrieden zu machen, um selbst im Wahlausgang erfolgreich zu sein. Ökonomen haben materielle Ressourcen hinsichtlich ihres Beitrages für die subjektive Zuf riedenheit lange überschätzt und gleichzeitig die Bedeutung nichtmaterieller Ressourcen wie soziale Beziehungen, Autonomie u.a. unterschätzt. Dass die Höhe des Einkommens nicht unbedingt positiv mit Lebenszufriedenheit korreliert, hängt u.a. auch mit psychologischen Prozessen der Veränderung des Anspruchs- (bzw. Vergleichs)-niveaus zusammen (Diener, Lucas, \& Scollon, 2006; Diener, 2008) (,hedonic treadmill”). Wenn man erlebt, dass es anderen ähnlich gut geht (durch allgemeine Wohlstandserhöhung), wird der eigene Wohlstand (Einkommenshöhe) nicht absolut sondem vielmehr relativ (z.B. im Vergleich zu anderen Personen) bewertet. Diese relative Bewertung kann auch in Bezug auf die eigene bisherige Einkommenshöhe in der Vergangenheit erfolgen. An ein höheres Einkommen gewöhnt man sich. Diese „Anpassung“ führt zu höheren Einkommenserwartungen und daher gerade nicht zu höherer Zufriedenheit (Komadt, in Druck; Trommsdorff, in Druck)

Die bei diesen psychologischen Prozessen wirksame Rolle des Vergleichsstandards ist seit der frühen experimentellen Psychologie (Helson, 1964), in der frühen Entscheidungs- und Leistungsmotivationsforschung (Atkinson, 1964; Heckhausen, 1991) sowie auch in der frühen sozialpsychologischen Austauschtheorie (Thibaut \& Kelley, 1959) bekannt. Es geht dabei um die erfahrungsbedingte Veränderbarkeit des Anspruchsniveaus, das wiederum individuelle Zielsetzungen und den erwarteten subjektiven Nutzen von Handlungen bestimmt.

Inzwischen wird auch in der psychologischen, soziologischen und ökonomischen Glücksforschung gesehen, dass nicht nur materielle sondem auch psychologische Ressourcen wie Autonomie, Selbständigkeit, Kontrolle, Faimess, Freizeit etc. die Lebenszufriedenheit beeinflussen. Hier seien als Beispiel die Untersuchungen zur Lebenszufriedenheit bei Selbstständigen im Vergleich zu nichtselbstständig Arbeitenden und die Bedeutung von sozialem Engagement für subjektive Lebenszuf riedenheit (auf Grundlage von SOEP Daten nach 1990) genannt (Frey, 2008): Soziales Engagement ist eine eindeutig nichtmaterielle Ressource und bedeutet den teilweisen Verzicht auf eigenen materiellen Nutzen. Intergenerationale Unterstützungsleistungen innerhalb der Familie erhöhen die Lebenszufriedenheit wie auch eigene kulturvergleichende Studien an deutschen, chinesischen und indonesischen erwachsenen Töchtern zeigen (Schwarz et al., 2009).

In der ökonomischen und soziologischen Glücksforschung wurden zudem lange Zeit nur die kognitiven Aspekte der Lebenszuf riedenheit erfasst. Auch in der Psychologie war das Interesse lange Zeit nur auf kognitive Aspekte gerichtet, bis neuerdings erkannt wurde, dass auch und gerade die emotionalen Aspekte 
des Erlebens einen wesentlichen Beitrag zur subjektiven Zufriedenheit leisten. Daher ist im SOEP vor kurzem eine eigene Skala eingefüht worden, um den emotionalen Aspekt der Lebenszufriedenheit zu erfassen. Schimmack, Schupp und Wagner (2008) haben bereits nachgewiesen, dass die emotionale Komponente der Lebenszufriedenheit andere Verhaltensimplikationen als die kognitive Komponente hat, also daher auch für Soziologen und Ökonomen von Interesse ist (vgl. Schupp \& Wagner, in diesem Band; Trommsdorff 2009a; in Druck).

Allerdings sind individuelle und kulturelle Differenzen in der sozialwissenschaftlichen und ökonomischen Lebenszufriedenheitsforschung bisher zu wenig beachtet worden (vgl. Komadt, in Druck). Neuerdings werden stabile Persönlichkeitsmerkmale wie die „Big Five“ und genetische Faktoren als Bedingungen individueller Differenzen in der Lebenszufriedenheit (auf der Grundlage eines interindividuell variierenden Positivitätsbias) angenommen (Caprara et al., 2009). Individuelle Differenzen in der Zufriedenheit stehen auch in Zusammenhang mit „Fehlern“ in der Zuschreibung von Ursachen für Verhalten bei anderen und bei sich selbst (interne oder externe, stabile oder variable Ursachen). Zufriedenheit wird zudem durch den (allerdings kulturspezifisch variierenden) Attribuierungsfehler beeinflusst (z.B. sich selbst eher positive Absichten und Fähigkeiten zuzuschreiben und weniger den anderen) (vgl. Trommsdorff, 2007b). Weiter haben sich Zusammenhänge zwischen Lebenszufriedenheit mit Zukunftsorientierung (Optimismus, Pessimismus), mit Kontroll - und Selbstwirksamkeitsüberzeugungen und Selbstregulation (u.a. der Fähigkeit und Bereitschaft zum Belohnungsaufschub; zur Persistenz bei der Zielverfolgung) (Bandura, 2001) gezeigt. Schließlich nehmen Ryan und Deci (2000) individuelle Unterschiede in der Zufriedenheit bei der Erfüllung der angenommenen universellen Grundbedürfnisse der Autonomie, Verbundenheit und Kompetenz an.

3.3 Systematische Urteilsfehler als Untersuchungsgegenstand der Psychologie und der Ökonomie: Implikationen für das Rationalitäts-Konzept (und rational choice)

Für Bruno Frey (2008) und seine Mitarbeiter bedeutet die Glücksforschung eine Revolution in der Ökonomie. Frey geht davon aus, dass das Konzept Glück oder Lebenszufriedenheit ein Proxi (Indikator) fiir das Konzept des Nutzens (utility) ist; Nutzen (äquivalent zu Präferenzen) ist in der Ökonomie eine zentrale Grundlage der klassischen Modelle von „rational choice“. Aus ökonomischer Sicht maximieren Individuen ihren Nutzen; sie verhalten sich nur zu fällig falsch bzw. nicht rational. Das allgemeine zugrunde liegende nutzentheoretische Modell lautet $(\mathrm{N}=\mathrm{E} \times \mathrm{W}-\mathrm{K})$. Der Nutzen $(\mathrm{N})$ wird also gesehen als Funktion von subjektiven Erwartungen von Zufriedenheit (Ertrag der Alternative) (E) multipli- ziert mit subjektiver Wahrscheinlichkeit, dass der Ertrag eintrifft (W) minus der Kosten für die Realisierung der Alternative (K). Bei einer so streng nutzentheoretischen Sichtweise werden allerdings ein mechanistisches Menschenbild und ein universalistisches Konzept von „Nutzen“ vorausgesetzt und individuelle Differenzen ignoriert

Psychologische Studien zeigen jedoch, dass individuelle Differenzen in der „Nutzenmaximierung“ erheblich sind: für verschiedene Menschen sind „Nutzen“ etwas ganz verschiedenes. Menschen aus verschiedenen Kulturen oder Sozialisationskontexten streben ganz verschiedene Ziele (mit sehr unterschiedlichen und keineswegs immer erfolgreichen Handlungen) an. Sie erleben das Erreichen verschiedener Ziele (z. T. je nach kulturellen Werten, oder je nach Wichtigkeit von Autonomie, Kompetenz und Verbundenheit) als unterschiedlich befriedigend. Dies hängt auch damit zusammen, dass weitere individuelle Faktoren (bzw. Persönlichkeitsmerkmale) wie Risikobereitschaft, Optimismus, Vertrauen, Unsicherheitsvermeidung u.a. eine Rolle spielen. Daher sind die frühen psychologischen Erwartungs-Wert-Theorien der Entscheidungsforschung inzwischen differenziert worden. Aus psychologischer Sicht machen Individuen systematisch „Fehler“ (eingeschränkte Rationalität). Die von Psychologen schon früh nachgewiesenen systematischen „Fehler" bei Wahrnehmung, Urteilsbildung und Entscheiden werden allmählich in der ökonomischen Forschung zur „Nutzenmaximierung" erkannt und in die Theoriebildung mit einbezogen.

Die oben skizzierten Bedingungen für individuelle Differenzen in der Lebenszufriedenheit sind Beispiele für solche systematischen „Urteils-Fehler“. Diese Fehler oder Verzerrungen sind teilweise bedingt durch Persönlichkeitsmerkmale, teilweise sind sie auch durch situative Bedingungen (und Rahmeneffekte, „framing") beeinflusst. In der Sozialpsychologie hat beispielsweise die frühe gruppendynamische Forschung gezeigt, dass situative Faktoren normative Wirkung haben können und so fehlerhafte Urteile bewirken. Einzelurteile tendieren angesichts von falschen, aber einstimmigen Gruppenurteilen dazu, mit dem falschen Gruppenurteil konform zu gehen (Ash, 1955). Von sozialen Gruppen gebildete Urteile sind unter bestimmten Bedingungen riskanter als Urteile von einzelnen, isoliert entscheidenden Gruppenmitgliedern (Kogan, Lamm, \& Trommsdorff, 1972). Die Eigengruppe (auch wenn sie künstlich gebildet wurde) wird gegenüber der Fremdgruppe positiver bewertet und für Kooperation bevorzugt (Sherif, 1956).

Des Weiteren können auch individuelle mentale Prozesse zu Verzerrungen und Fehlern führen (z.B. Stereotype; Anspruchs- und Vergleichsstandard). Mentale Repräsentationen von alternativen früheren Ereignissen (upward/downward counterfactual thinking) können gegenwärtige Emotionen, Wahmehmungen und Selbstbewertungen sowie Handlungen beeinflussen (Byme, 2005; McCrea, 
2008). Kahneman und Tversky (1982) haben solche mentalen Prozesse (zu „alternativen Realitäten") mit Folgen für Urteilsverzerrungen und ihrem Einfluss auf Entscheidungen (Kaufentscheidungen, Beruf splanungen) untersucht. In weiteren Studien zu Urteilsheuristiken, Risikoentscheidungen und Framing-Ef fekten im Zusammenhang mit dem Konzept der „,bounded rationality“ (Simon, 1979) differenziert Kahneman (2003) verschiedene Aspekte von Urteilsverzerrungen. Ein weiteres Beispiel sind die Arbeiten von Gigerenzer (2008) zu Urteilsheuristiken, in denen ähnlich wie bei Gilovich, Griffin und Kahnemann (2002) u.a. intuitive (quasi-automatische) Urteile (intuitive statistische Inferenzen) (im Widerspruch zu statistischen Prinzipien) nachgewiesen werden. Diese Befunde veranlassen Ariely (2008) zu dem Schluss, dass Verzerrungen und Illusionen nicht nur die Wahrnehmung und Urteilsbildung beeinflussen sondern auch unsere Entscheidungen (,decision illusions").

Damit ergibt sich für die Sozial- und Wirtschaftswissenschaften sowie die Psychologie die Forschungsfrage nach den Bedingungen von Urteilsverzerrungen. Hier sind zum emen mdividuelle Unterschiede in der Bevorzugung von bestimmten Verzerrungen zu berücksichtigen (z.B. illusionäre Kon rollüberzeugung), sowie zum anderen situative Einflüsse (z.B. Zeit- und Gruppendruck) und nicht zuletzt signifikante kulturelle Unterschiede (z.B. vermittelt über Werthaltungen).

Zusammenfassend ist wirtschaftliches wie auch anderes soziales Handeln ven einer Vielfălt affektiv getönter mentaler Prozesse wie Vertrauen, Reziprozität, Fairness beeinflusst. Diese psychologischen Prozesse stehen scheinbar im Widerspruch zur klassischen Rationalitätsannahme der Ökonomie. In den Sozialund Wirtschaftswissenschaften sind psychologische Befunde, dass Gruppenprozesse, soziale Interaktionen und individuelle emotionale, motivationale und kognitive Prozesse zu Urteilsverzerrungen führen und Entscheidungsverhalten sowie auch die Lebenszufriedenheit beeinflussen können, teilweise aufgegriffen und experimentell untersucht worden. Inzwischen erscheinen „Fehler“ des vermeintlich nicht rational urteilenden bzw. handelnden Menschen als ganz „normal“ und sogar als Bedingungen für Lebenszufriedenheit. Wirtschaftshandeln ist wie auch anderes soziales Handeln durch vielfältige sonstige kognitive, motivationale und emotionale Prozesse beeinflusst. Dies legt eine psychologisch fundierte Differenzienung der klassischen Annahme rationalen Handelns unter Einbeziehung kultureller Faktoren nahe.

Zwar kann die Selektion psychologischer Konzepte ohne ausreichende Berücksichtigung der theoretischen und methodischen Grundlagen auch zu vereinfachenden Schlüssen führen. Jedoch hat die ökonomische und sozialwissenschaftliche Forschung inzwischen ausgewählte psychologische Forschung für eigene Fragestellungen und Theorien nutzbar gemacht.
4 Relevanz sozial-und wirtschaf tswissenschaftlicher Forschung für Psychologie

4.l Was die Psychologie von den Sozial- und Wirtschaftswissenschafien gelernt hat

Sozialwissenschaftliche Indikatoren, Methoden und Datensätze. Die Sozialwissenschaften haben u.a. insofern einen wichtigen Beitrag fuir die psychologische Forschung geleistet, als in der Psychologie erkannt wurde, dass bei Interesse an generalisierbaren empirischen Ergebnissen sozio-strukturelle Variablen zu berücksichtigen sind. In entwicklungs- und sozial psychologischen Untersuchungen ist daher inzwischen üblich, dass soziodemografische Merkmale der Untersuchungsteilnehmer mit erfasst werden. Allerdings bleibt es zumeist bei Hinweisen auf SES (den sozio-ökonomischen Status), der überwiegend aufgrund des Bildungsabschlusses der Eltern ermittelt wird. Einkommen und Beruf etc. bleiben meistens unberücksichtigt, obwohl seit Kohn (1984) bekannt ist, dass u.a. gerade die Berufstätigkeit der Eltern ein wichtiger Sozialisationsfaktor für die Kinder ist, u.a. in Bezug auf deren Autonomie. Die neueren soziologischen Studien zur Spezifizierung des Berufsstatus (Müller, 2008) mit dem Ziel für vergleichende Analysen funktional äquivalente Indikatoren zu bilden, werden in der Psychologie bisher noch nicht zur Kenntnis genommen.

Die thematischen und methodischen Möglichkeiten psychologischer Forschung wurden zudem signifikant erweitert, weil Daten des Sozio-oekonomischen Panel (SOEP) für Referenzstichproben genutzt werden und weil spezifische SOEP-Stichproben in Zusammenhang mit nicht-repräsentativen Daten psychologischer Studien gebracht werden können; dies erlaubt u.a. auch, die bislang fehlenden Indikatoren für sozialwissenschaftlich relevante Variablen (wie Berufsstatus, Mobilität etc.) in Analysen zur Entwicklung über die Lebensspanne mit einzubeziehen.

Thematische Erweiterungen. Für inhaltliche Fragen zur Kontinuität und Veränderung von individuellen Einstellungen und Verhalten über die Lebensspanne ergeben sich bislang verschlossene Möglichkeiten für vergleichende Analysen von Entwicklungsverläufen in sich verändernden sozialen Kontexten (Trommsdorff, 2008a). Datensätze aus den 25 Jahren, die das SOEP existiert, stellen inzwischen eine für die Psychologie wertvolle Datenquelle dar. So wurde z.B. durch Initiative des SOEP unmittelbar nach der Wende eine repräsentative Erhebung von Haushaltsmitgliedern (u.a. auch zu psychologischen Variablen wie Lebenszufriedenheit, Optimismus, Kontrollorientierung) in Ostdeutschland durchgefuihrt; dieser folgten dann die regelmäßigen Befragungen im Rahmen des Panel (vgl. Wagner et al., 2008; Schupp \& Wagner, in diesem Band). Diese Daten sind gerade für Psychologen besonders interessant, nachdem 20 Jahre nach 
der Wende die damaligen Kinder und Jugendlichen heute selbst erwachsen sind und ihrerseits in die Befragungen mit einbezogen werden.

Die Psychologie hat einerseits durch diese thematischen und methodischen Enxeitenungen profitiert. Andererseits kann sie auf Grundlage solcher Datensätze einen Beitrag leisten, der wiederum für die sozialwissenschafțliche Forschung zum sozialen Wandel von Bedeutung ist. Hier sehen wir ein Beispiel gegenseitiger Befruchtung sozialwissenschaftlicher und psychologischer Forschung.

\subsection{Wodurch psychologische Forschung gesellschafitliche Relevanz gewinnen kann}

Die Wirtschafts- und Sozialwissenschaften haben u.a. zum einen dazu beigetragen, psychologische Forschung zu bestimmten Themen und Fragen anzuregen, wie auch umgekehrt die psychologische Forschung verschiedene Fragestellungen, Theorien und Methoden der Sozialwissenschaften und Verhaltensökonomie beeinflusst hat. Bemerkenswert ist der neuerdings sichtbare Beitrag psychologischer Forschung für gesellschaftspolitische Fragen, der ohne die Vermittlung durch die Wirtschafts- und Sozialwissenschaften nicht möglich wäre.

Ein Beispiel für eine solche gegenseitige Befruchtung mit Folgen für sozialpolitische Diskussionen sind die inzwischen von den Sozial- und Wirtschaftswissenschaften rezipierten entwicklungspsychologischen Erkenntnisse zu Lang zeitefjekten von frühkindlicher Entwicklung. So hat sich die frühe Förderung der Entwicklung von Kindenı als ein wichtiger Faktor für die spätere Entwicklung von Interessen, Lemmotivation und sozialer Kompetenzen mit lebenslangen Wirkungen für Bildungs- und die Berufskarriere erwiesen. Diese wiederum bedeuten eine wichtige sozio-ökonomische Ressource (vgl. Heckman, 2006). Dabei waren es weniger die entwicklungspsychologischen Studien selbst, sondern vielmehr haben erst die Arbeiten der Ökonomen von Heckman und Kollegen die kumulativen wirtschaftlichen Konsequenzen unzureichender frühkindlicher Erziehung aufgezeigt. Diese Rezeption hat nicht nur dazu beigetragen, dass entwicklungspsychologische Forschung in ökonomisch orientierte Panelstudien (wie in das SOEP) eingegangen ist. Darüber hinaus werden diese psychologischen Erkenntnisse in der Öffentlichkeit als volkswirtschaftlich und sozialpolitisch relevant diskutiert und als Begründung für bildungs- und sozialpolitische Maßnahmen verwendet. Die sozialpolitischen Folgen zeigen sich u.a. darin, dass in Deutschland die frühkindliche Erziehung plötzlich ein öffentliches Thema ist und gefordert wird.

Ein weiteres Beispiel gegenseitiger Befruchtung zwischen Psychologie und Wirtschafts- und Sozialwissenschaften mit potentiellen sozialpolitischen Folgen zeigt sich u.a. auch darin, dass die Ökonomie die Bedeutung von sogenannten „,nichtkognitiven“ Fähigkeiten wie der Selbstregulation als Ressource und deren Entwicklungsbedingungen erkannt hat und inzwischen selbst untersucht. Unter Selbstregulation hat man aus psychologischer Sicht sowohl die Fähigkeit als auch die Bereitschaft zu verstehen, vor allem aktuelle Impulse und Bedürfnisse zu kontrollieren. Dies ist speziell beim Lemen relevant sowie auch in sozialen Interaktionen. Selbstregulation ist nach ihren verschiedenen Komponenten zu differenzieren. Dazu gehören unter anderem Auf rnerksamkeitslenkung, Verhaltenskontrolle (wie Belohnungsaufschub) (Mischel \& Ayduk, 2004) sowie die Regulation eigener Emotionen jeweils für verschiedene Handlungsbereiche (Trommsdorff, 2009c; Trommsdorff \& Cole, in Druck; Suchodoletz, Trommsdorff, Heikamp, Wieber, \& Gollwitzer, in Druck).

Individuelle Differenzen in ,nichtkognitiven“ Fähigkeiten der Selbstregulation entstehen aufgrund bestimmter Sozialisationsbedingungen in Familie und Schule und wirken sich auf verschiedene Verhaltensbereiche aus. In einem größeren Forschungsprojekt untersuchen wir gegenwärtig, inwieweit die Selbstregulation im Vorschulalter die Entwicklung im Schulalter vorhersagen kann, und zwar sowohl in Bezug aufSchulleistungen als auch in Bezug auf Sozialverhalten (Trommsdorff, 2008b)

Die Bedeutung der Selbstregulation geht vermutlich über ihre Rolle für schulische und soziale und emotionale Kompetenz hinaus und ist fur andere Bereiche wie Gesundheitsverhalten, gesellschaftliche und politische Partizipation anzunehmen. Komponenten der Selbstregulation wie Belohnungsaufschub und Frustrationstoleranz sind Bedingungen für Bildungsinvestitionen. Wenn Selbstregulation zum Beispiel den Schulerfolg fördert (und Schulabbruch verhindert) und damit Voraussetzungen fürr spätere Unabhängigkeit von elterlicher oder staatlicher Versorgung vermittelt, belegt dies ihre bildungs-, gesellschafts- und wirtschaftspolitische Relevanz.

Sozial- und Wirtschaftswissenschaften können somit „Entwicklungshilfe“ für die Sichtbarkeit und Wirkung dieser psychologischen Forschungen leisten und zusammen mit der Psychologie an Grundlagen für solide gesellschaftspolitische Maßnahmen mitwirken.

\subsection{Grenzen und Möglichkeiten gegenseitigen Nutzens bei Einbeziehung} kulturinformierter Forschung

Eine Abgrenzung von Sozialwissenschaften und Psychologie ist jedoch weiterhin unvermeidlich, weil beide einen verschiedenen Gegenstandsbereich untersuchen. Dennoch zeigen sich zahlreiche Möglichkeiten fruchtbarer gegenseitiger theoretischer und methodischer Anregungen. Dies wird besonders deutlich in der kulturvergleichenden Psychologie, wenn sie kulturpsychologische Phänomene an 
den Schnittstellen zwischen Individuum und Gesellschaft untersucht (vgl. Kornadt, in diesem Band; Eckensberger, in diesem Band; Schwarz, in diesem Band; See \& Essau, in diesem Band; Friedlmeier, in diesem Band; Grossmann \& Grossmann, in diesem Band; Rothbaum \& Wang, in diesem Band).

Dabei entstehen universelle Fragen nach den Grundlagen der Beziehungen zwischen Individuum und sozialer Gruppe, u.a. unter Berücksichtigung von Autonomie und Verbundenheit in der Entwicklung des Einzelnen, und unter Berücksichtigung sozialer Sukturen wie Gleichheit und Hierarchie sowie der Art sozialer Beziehungen (Konflikt oder Harmonie). Dabei können kulturelle Werthaltungen als relativ stabile individuelle Orientierungen die Funktion der Herstellung von Kohärenz in Gesellschaften haben. Werte sind zum einen auf der kulturellen sowie zum anderen auf der individuellen Ebene wirksam. Daher können Werte zur Analyse von Kulturdimensionen sowie von individuellen Handlungsorientierungen dienen und als Bindeglied zwischen Makro-, Mikro- und individueller Ebene fungieren (Schwartz, 2004; Trommsdorff, 2007a; 2009c).

Auch wenn in der Psychologie gegenwärtig eher eine naturwissenschaftliche Sichtweise dominiert und eine sozial- oder gar kulturwissenschaftliche Orientierung keinen leichten Stand hat, erscheint es besonders wichtig, die Analyse aktueller gesellschaftlicher Probleme nicht nur der Soziologie oder den Wirtschaftswissenschaften zu überlassen sondern zu versuchen, auch aus kulturinformierter psychologischer Sicht einen Beitrag zu leisten. Damit lässt sich vielleicht die von Wilhelm Wundt diskutierte Dualität der Psychologie in einem anderen Licht sehen (vgl. Graumann, 2006; Kornadt, in diesem Band).

Insbesondere in der kulturvergleichenden Psychologie, der Sozialisationsforschung und der Entwicklungspsychologie ist die Berücksichtigung mehrerer Analyseebenen unverzichtbar. Die Zusammenhänge zwischen Makro-, Mikround individueller Ebene sind allerdings bisher nicht ausreichend geklärt. Jedoch haben Teile der heutigen Sozialwissenschaften, einschließlich der Psychologie heute erkannt, dass es erforderlich ist, die verschiedenen Ebenen menschlichen und sozialen Handelns jeweils zu berücksichtigen und den schwierigen Schritt zur Verknüpf ung dieser verschiedenen Ebenen zu versuchen.

\section{Zusammenf assung und Ausblick}

5.1 Gemeinsame methodologische Probleme von Psychologie und Sozialwissenschaften

Ähnlich wie die Sozialwissenschaften hat sich die Psychologie mit der wissenschaftstheoretischen Frage befasst, ob sich auf die Psychologie die gleichen Regeln anwenden lassen wie auf die Naturwissenschaften. Auch wenn der von
Karl R. Popper (1935) vertretene kritische Rationalismus als Grundlage empirisch fundierter Naturwissenschaften (Albert, 1969) inzwischen weitgehend akzeptiert ist, stellt sich jedoch für die Sozialwissenschaften und die Psychologie die Frage, ob angesichts verschiedener Besonderheiten dieser Disziplinen (z.B. deren sozio-kulturelle Verankerung) überhaupt ein einheitlicher theoretischer und methodologischer Zugang angemessen ist (Esser, 2008).

So werden in den Sozialwissenschaften und in der Psychologie die Möglichkeiten und Probleme von universellen (ahistorischen und überkulturellen) Gesetzen, der Trennung von deskriptiven und normativen Aussagen und der Sicherung von Objektivität scheinbar objektiver empirisch geprüfter Aussagen diskutiert. Diese Probleme haben kein Äquivalent in den Naturwissenschaften.

Wenn allein die Ebene des Individuums oder allein die der Makro- oder Mesoebene unzureichend für die jeweils gesuchten Erklärungen sind, stellt sich die Forderung, diese Ebenen systematisch in Zusammenhang miteinander zu untersuchen. Allerdings besteht das Problem, dass diese verschiedenen Analyseebenen jeweils eigenen methodologischen Regeln folgen. Dabei sind sowohl die Probleme der hermeneutischen Interpretation individuellen Handelns als auch die Probleme der Erklärung einer Transformation von nichtintendierten und emergenten Folgen individuellen Handelns in Kollektiven zu berücksichtigen. Dies ist schwierig, wenn die sozialen Prozesse spezifische Emergenzen auf weisen, ,....die sich womöglich auf das Handeln individueller Akteure und nomologische Theorien über sie nicht reduzieren lassen“ (Esser, 2008, S. 1493). „Unter Emergenz verstehen wir heute, systemtheoretisch gesprochen, das „Auftauchen einer Entität oder Eigenschaft, die in den sie konstitutierenden Elementen (noch) nicht existierte." (Graumann, 2006, S. 60).

Soziale Strukturen lassen sich nicht schlicht auf individuelles Handeln zurückfïhren. Auch aggregierte Daten individueller Akteure können nicht Phänomene sozialer Strukturen beschreiben. Individuelle Handlungen können jedoch vermittelt durch soziale Interaktionen zu sozialen Phänomenen führen. So können soziale Normen und Rollenzuweisungen entstehen, die durch Sanktionen verfestigt und für den Einzelnen handlungswirksam werden. Diese können unerwünschte Verhaltensänderungen bewirken, wie z.B. die Bereitschaft zu extremeren (z.B. riskanteren) Urteilen fördern und Aggression und Gewalt gegenüber Einzelnen plötzlich legitimieren (Zimbardo, 2007).

In der kulturvergleichenden Forschung ist die Berücksichtigung verschiedener Analyseebenen unverzichtbar. Mehrere Ebenen werden in der ökologischen Systemtheorie von Bronfenbrenner $(1979,1989)$ differenziert; die Makro-, Meso- und Mikroebene wird durch eine Chrono-Ebene ergänzt. Allerdings bedarf es für methodisch abgesicherte Untersuchungen auf diesen Ebenen relativ komplexer Vorgehensweisen. So sollten die Analysen der (auf Individual- und Gruppenebene hierarchisch geschachtelten und voneinander abhängigen) Daten durch 
Mehrebenenmodelle abgesichert werden, so dass kulturelle und individuelle Variablen angemessen berücksichtigt werden (vgl. Van de Vijver, van Hemert, \& Poortinga, 2008). Dass dies fruchtbar ist, zeigen u.a. Analysen zum Zusammenhang zwischen Kulturmerkmalen und Kinderwunsch im Rahmen der VOCStudie (Mayer, 2009; Mayer \& Trommsdorff, 2009).

Grundsätzlich sind verschiedene methodologische Anforderungen an makro- und mikrosoziologische und psychologische Analysen zu stellen. Des Weiteren muss die wissenschaftstheoretisch wichtige Frage nach dem Ausgangspunkt der Theoriebildung im Auge behalten werden, also die individuellen Akteure (bzw. deren Handlungen) oder gesellschaftliche Systeme.

\subsection{Beziehungen zwischen Psychologie und Sozialwissenschaften. Gegenseitiger Nutzen}

Dieses Kapitel sollte die Beziehungen zwischen Psychologie und Sozialwissenschaften mit Blick auf den möglichen gegenseitigen Nutzen zeigen. Die Sozialwissenschaften befassen sich mit der Beschreibung und Erklärung gesellschaftlicher Phänomene und Probleme, die für die Psychologie von unmittelbarem Interesse sind. Erkenntnisse der psychologischen Grundlagen- und angewandten Forschung können ihrerseits einen Beitrag zur Bearbeitung sozialwissenschaftlicher Fragen leisten, wie einige hier diskutierte Beispiele zeigen.

Eine Herausforderung sowie ein deutlicher Gewinn für die Psychologie ist gegeben, wenn sich psychologische und sozialwissenschaftliche Ansätze so miteinander verbinden lassen, dass psychologische Phänomene im Zusammenhang mit dem gegebenen sozio-kulturellen Kontext erklärt werden können. Dies ist in der Forschung zum sozio-ökonomischen und demografischen Wandel und in der kulturvergleichenden Psychologie vielfach der Fall. Des Weiteren besteht ein Gewinn fur die psychologische Forschung darin, sich sozialwissenschaftlich erprobter Indikatoren und Methoden bedienen zu können.

Ein Gewinn für die Sozial- und Wirtschaftswissenschaften ist darin zu sehen, wenn sozial- und wirtschaftswissenschaftliche Phänomene im Zusammenhang mit psychologischen Prozessen erklärt werden können. Für strukturelle und makrosoziologische Analysen sind jedoch allenfalls psychologische Ansätze als Analogien verwendbar. Ein Gewinn durch Einbeziehung psychologischer Ansätze und Methoden hat sich für mikrosoziologische Analysen z.B. zum Wandel von Sozialisationsbedingungen, Familienformen, und den Lebenslauf gezeigt. Für sozialwissenschaftliche und verhaltensökonomische Analysen hat sich ein Gewinn u.a. durch die Berücksichtigung emotions-, motivations- und kognitionspsychologischer Erkenntnisse, sowie durch Forschung zu Persönlichkeits- merkmalen und bestimmten Verhaltensbereichen wie Altruismus, Kooperation und Entscheidungen enwiesen. Ein Beispiel fuir den Einfluss der Psychologie auf die Ökonomie und Soziologie sind die inzwischen kritisch diskutierten Annahmen zu Rationalität menschlichen Verhaltens (u.a. Entscheidungsverhalten). Durch umfangreiche psychologische Forschung über ,normale“, systematische „Fehler" bei Urteilen und Entscheidungen ist inzwischen ein Umdenken in der verhaltenswissenschaftlich orientierten Ökonomie entstanden. Inzwischen haben einige Bereiche der Sozialwissenschaften und Verhaltensökonomie psychologische Ansätze und Methoden (Experimente) für die Bearbeitung eigener Fragestellungen erfolgreich angewendet. Auch hinsichtlich der Operationalisierung von Konzepten hat die Psychologie einen Beitrag fiir sozialwissenschaftliche Untersuchungen geleistet; dies wird am Beispiel der im SOEP erfolgten Erweiterung der Bef ragungen durch psychologische Methoden deutlich.

Eine gegenseitige Bereicherung in der Zusammenarbeit von Psychologie und den Sozialwissenschaften wird deutlich, wenn psychologische Phänomene und Prozesse im sozio-kulturellen Kontext untersucht werden, also z.B. wenn es um Fragen der Universalität oder Kulturspezifität psychologischer Prozesse oder um die Aufklärung von Bedingungen für gesellschaftlich relevantes Verhalten wie pro- und antisoziales oder abweichendes Verhalten, Schulerfolg, Familiengründung oder Migration geht. Diesen Fragen liegt die Annahme zugrunde, dass ein Erkenntnisgewinn in der psychologischen Forschung u.a. von der Berücksichtigung von biologischen und psychologischen Merkmalen der Person und von Kontextfaktoren und von deren Zusammenwirken abhängt.

Die direkte Zusammenarbeit zwischen Psychologen und Soziologen, wie sie heute in größeren kulturvergleichenden Projekten erfolgt, hat eine Ausweitung der thematischen, theoretischen und methodischen Grundlagen für beide Disziplinen ermöglicht. So haben Psychologen z.B. im Rahmen des Projektes „Value of Children and Intergenerational Relations" durch die Zusammenarbeit mit Soziologen neue Ansätze zur Erfassung von Merkmalen sozialer Integration, der Familienstruktur etc. (z.B. durch Netzwerkanalysen) und von Merkmalen sozioökonomischen Wandels (z.B. durch Indikatoren auf Makroebene) gewonnen, die für die Bearbeitung kulturvergleichender psychologischer Fragen notwendig sind (Trommsdorff, 2009b; Trommsdorff \& Nauck, 2005; 2006; 2009; Mayer \& Trommsdorff, 2009)

Für die zukünftige Forschung bestehen für beide Disziplinen noch höchst umfangreiche und vielversprechende Aufgaben. Diese betreffen u.a. auch die hier nicht weiter ausgefuihrte Rolle der biologischen Bedingungen individuellen und sozialen Handelns (z.B. in der Empathie, der Transmission von kulturellen Ressourcen) (vgl. Tomasello, 2009). Während sich die Psychologie mit den biologischen Grundlagen individuellen Handelns zunehmend intensiver befasst 
(vgl. Übersicht bei Diamond, 2009), zudem ihre naturwissenschaftlichen Wurzeln dies als eine wichtige Ausrichtung erfordern, besteht für die Sozialwissenschaften hier weiterhin ein erheblicher Nachholbedarf.

Mit der Neuroökonomie beginnt inzwischen eine Neuorientierung in den Wirtschaftswissenschaften. Für die damit verbundenen komplexen Forschungsaufgaben ist es unvermeidlich, komplexere Modelle menschlichen Verhaltens und gesellschaftlicher Systeme zugrunde zu legen. Dabei muss über statische lineare Modelle hinausgegangen und das Potential nichtlinearer dynamischer Modelle (trotz damit verbundener neuer Probleme) genutzt werden (Efferson \& Richardson, 2007).

Eine gegenseitige Bef ruchtung zwischen den Disziplinen ist besonders gut möglich z.B. auf Gebieten wie der Bedeutung von biologischen und Umweltbedingungen in der Sozialisation und der Entwicklung über eine verlängerte Lebensspanne; ebenso in Bezug auf die sich wandelnden sozio-ökonomischen und kulturellen Systeme. Ein Beispiel wäre die Wirkung des Klimawandels auf individuelles und soziales Handeln unter verschiedenen sozio-ökonomischen Bedingungen.

Zusammenfassend ist zu erwarten, dass die zukünftigen Beziehungen zwischen psychologischer und sozialwissenschaftlicher Forschung vor neuen Herausforderungen stehen, um wichtige Beiträge zur Aufklärung menschlichen Handelns leisten zu können. Die kulturvergleichende Psychologie kann eine Brücke zwischen psychologischen und sozio-ökonomischen Fragestellungen bilden. Sie kann einerseits von der sozio-ökonomischen Forschung profitieren, wenn es z.B. um die Berücksichtigung der strukturellen Kontextbedingungen und ihrer Funktionen geht. Andererseits würde die sozio-ökonomische Forschung durch die bisher unzureichende Berücksichtigung psychologischer, kulturbedingter Prozesse des sozialen Handelns bereichert werden.

\section{Literatur}

Albert, H. (1969). Traktat über kritische Vernunft. Tübingen: Mohr.

Albert, 1. (2007). Intergenerationale Transmission von Werten in Deutschland und Frankreich. Lengerich: Pabst Science.

Ariely, D. (2008). Predictably irrational: The hidden forces that shape our decisions. London: HarperCollins.

Arsenio, W. F. (2008). Psychological limits of economic rationality: Relational contexts and cognitive irrationality: Commentary on Gummerum, Hanoch and Keller. Human Development, 51, 268-273.

Asendorpf, J. B. (2008). Living apart together: Alters- und Kohortenabhängigkeit einer heterogenen Lebensform. Kölner Zeitschrift für Soziologie und Sozialpsychologie, 60, 749-764.

Ash, S. E. (1955). Opinions and social pressure. Scientific American, 193, 31-35.
Atkinson, J. W. (1964). An introduction to motivation. Princeton, NJ: Van Nostrand.

Bandura, A. (2001). Social cognitive theory: An agentic perspective. Annual Review of Psychology, 52(1), 1-26.

Bengtson, V. L. (2001). Beyond the nuclear family: The increasing importance of multigenerational bonds (The Burgess Award Lecture). Journal of Marriage \& the Family, 63(1), 1-16

Berry, J. W., Poortinga, Y. H., \& Pandey, J. (Eds.). (1997). Handbook of cross-cultural psychology: Vol. 1. Theory and method. Boston: Allyn \& Bacon.

Bertram, H., Nickel, H. M., Niedermayer, O., \& Trommsdorff, G. (Hrsg.). (2006). Berich te zum sozialen und politischen Wandel in Ostdeutschland (6 Bände). Opladen: Leske und Budrich.

Bischof-Köhler, D. (1991). The development of empathy in infants. In M. E. Lamb \& H. Keller (Eds.), Infant development: Perspectives from German-speaking countries (pp. 245-273). Hillsdale, NJ: Erlbaum

Bischof-Köhler, D. (2000). Kinder auf Zeitreise. Theory of mind, Zeitverständnis und Handlungsorganisation. Bern: Hans Huber.

Bronfenbrenner, U. (1979). The ecology of human development: Experiments by nature and design. Cambridge, MA: Harvard University Press.

Bronfenbrenner, U. (1989). Ecological systems theory. Annals of Child Development, 6 , $187-249$.

Byme, R. M. (2005). The rational imagination: How people create alternatives to reality. Cambridge, MA: MIT Press.

Caprara G. V, Fagnani, C., Alessandri, G., Steca, P., Gigantesco, A., Cavalli Sforza, L.L. \& Stazi, M. A. (2009). Human optimal functioning: The genetics of positive orientation towards self, life, and the future. Behaviour Genetics, 39, 277-284.

Chen, X., \& Chen, H. (in press). Children's socioemotional functioning and adjustment in the changing Chinese society. In R. K. Silbereisen \& X. Chen (Eds.), Social change and human development: Concepts and results. London: Sage Publications.

Dahrendorf, R. (1957). Soziale Klassen und Klassenkonflikt in der industriellen Gesellschaft. Stuttgart: Enke.

Dahrendorf, R. (1965). Gesellschaft und Demokratie in Deutschland. München: Piper.

Dasen, P. (2007). Ein integrativer theoretischer Rahmen menschlicher Entwicklung aus öko-kultureller Perspektive. In G. Trommsdorff \& H.-J. Kornadt (Hrsg.), Enzyklopädie der Psychologie: Themenbereich C. Theorie und Forschung: Serie VII. Kulturvergleichende Psychologie: Band 1. Theorien und Methoden in der kulturvergleichenden und kulturpsychologischen Forschung (S. 531-554). Göttingen: Hogrefe.

Dawkins, R. (1976). The selfish gene. Oxford: Oxford University Press.

Diamond, A. (2009). The interplay of biology and the environment broadly defined. Developmental Psychology, 45, 1-8.

Diener, E. (2008). Myths in the science of happiness, and directions for future research. In M. Eid \& R. J. Larsen (Eds.), The science of subjective well-being (pp. 493-514). New York: The Guilford Press.

Diener, E., Lucas, R. E., \& Scollon, C. N. (2006). Beyond the hedonic treadmill: Revising the adaptation theory of well-being. American Psychologist, 61, 305-314. 
Durkheim, E (1933). The division of labor in society. Translated by George Simpson. Ncw York: The Frec Press.

Eckensberger, L. H. (2008). Epilog. Dank - Rückblick - Diskussion. In I. Plath (Hrsg.), Kultur - Handlung -.- Demokratie (S. 195-242). Wiesbaden: VS Verlag für Sozialwissenschaften.

Efferson, C., \& Richardson, P. J. (2007). A prolegomenon to nonlinear empiricism in the human behavioral sciences. Biology and Philosophy, 22, 1-33.

Eisenberg, N. (2002). Empathy-rclated emotional responses, altruism, and their socialization. In R. J. Davidson \& A. Harrington (Eds.), Visions of compassion: Western scientists and Tibetan Buddhists examine human nature (pp. 131-164). London: Oxford University Press.

Eisenberg, N., Fabes, R. A., \& Spinrad, T. L. (2006). Prosocial development. In W. Damon, R. M. Lemer \& N. Eisenberg (Eds.), Handbook of child psychology. Social, emotional. and personality development (pp. 646-718). New York: Wiley.

Elder, G. H., Jr. (1974). The children of the great depression: Social change and life experience. Chicago: University of Chicago Press.

Elder, G. H., Jr. (1998). The life course as developmental theory. Child Development, 69, $1-12$.

Engeser, S., Rheinberg, F., \& Möller, M. (2009). Achievement motive imagery in Gernan schoolbooks: A pilot study testing McClelland's hypothesis. Journal of Research in Personality, 43, 110-113.

Esser, H. (2008). Wissenschaftstheorie der Sozialwissenschaften. In S. Gosepath, W. Hinsche, \& B. Rössler (Hrsg.), Handbuch der politischen Philosophie und Sozialphilosophie (S. 1490-1495). Berlin und New York: De Gruyter.

Fehr, E., \& Fischbacher, U. (2005). The economics of strong reciprocity. In H. Gintis, S. Bowles, R. Boyd, \& E. Fehr(Eds.), Moral sentiments and material interest. The foundations of cooperation in economic life (pp. 151-192). Cambridge, MA: MIT Press.

Frey, B. S. (2008). Happiness: A revolution in economics? Cambridge, MA: The MIT Press.

Friedlmeier, W., \& Matsumoto, D. (2007). Emotion im Kulturvergleich. In G. Trommsdorff \& H.-J. Komadt (Hrsg.), Enzyklopädie der Psychologie: Themenbereich C. Theorie und Forschung: Serie VII. Kulturvergleichende Psychologie: Band 2. Erle. ben und Handeln im kulturellen Kontext (S. 219-281). Göttingen: Hogrefe.

Gerhardt, U. (2009). Soziologie im zwanzigsten Jahrhundert. Studien zu ihrer Geschichte in Deutschland. Stuttgart: Franz Steiner Verlag.

GESIS Gesellschaft SoziaIwissenschaftlicher Infrastruktureinrichtungen (Hrsg.). (2008). Datenreport 2008. Ein Sozialbericht für die Bundesrepublik Deutschland. Bonn: Statistisches Bundesamt.

Gigerenzer, G. (2008). Rationality for mortals: How people cope with uncertainty. New York: Oxford University Press.

Gilovich, T., Griffin, D. W.. \& Kahneman, D. (Eds.). (2002). Heuristics and biases: The psychology of intuitive judgment. Cambridge: University Press.

Gintis, H., Bowles, S., Boyd, R., \& Fehr, E. (2005). Moral sentiments and material interests: Origins, evidence, and consequences. In H. Gintis, S. Bowles, R. Boyd, \& E.
Fehr (Eds.), Moral sentiments and material interests. The foundations of cooperation in economic life (pp. 1-39). Cambridge: The MIT Press.

Graumann, C. F. (2006). Die Verbindung und Wechselwirkung der Individuen im Gememschaftsleben. In G. Jüttemann (Hrsg.), Wilhelm Wundts anderes Erbe. Ein Missverständnis löst sich auf (S. 52-80). Göttingen: Vandenhoeck \& Ruprecht.

Heckhausen, H. (1991). Motivation and action (P. K. Leppmann, Trans.). Berlin, Heidelberg: Springer.

Heckman, J. J. (2006). Skill formation and the economics of investing in disadvantaged children. Science, 315, 1900-1902.

Helf rich, H. (2007). Sprachliche Kommunikation im Kulturvergleich. In G. Trommsdorff \& H.-J. Kornadt (Hrsg.), Enzyklopädie der Psychologie: Themenbereich C Theorie und Forschung, Serie VII Kulturvergleichende Psychologie. Band 2: Kulturelle De. terminanten des Erlebens und Verstehens (S. 109-156). Göttingen: Hogref c.

Helmke, A. (2008). Unterrichtsqualität und Lehrerprofessionalität. Diagnose, Evaluation und Verbesserung des Unterrichts. Seelze: Kallmeyer.

Helson, H. (1964). Adaption-Level Theory: An experimental and systematic approach to behavior. New York: Harper \& Row.

Hesse, H.-G. (2007). Lernen innerhalb und außerhalb der Schule aus interkultureller Perspektive. In G. Trommsdorff \& H.-J. Komadt (Hrsg.), Enzyklopädie der Psychologie: Themenbereich C Theorie und Forschung, Serie VII Kulturvergleichende Psychologie. Band 3: Anwendungsfelder der kulturvergleichenden Psychologie (S. 187-277). Göttingen: Hogrefe.

Hormuth, S. E., Heinz, W. R., Kornadt, H.-J., Sydow, H., \& Trommsdorff, G. (Hrsg.). (1996). Berichte zum sozialen und politischen Wandel in Ostdeutschland: Berichte der Kommission für die Erforschung des Sozialen und Politischen Wandels in den Neuen Bundesländern e.V. (KSPW): Bd. 4. Individuelle Entwicklung, Bildung und Berufsverläufe. Opladen: Leske und Budrich.

Kahneman, D. (2003). A perspective on judgment and choice: Mapping bounded rationality. American Psychologist, 58, 697-720.

Kahneman, D., \& Tversky, A. (1982). On the study of statistical intuitions. In D. Kahneman, P. Slovic, \& A. Tversky (Eds.), Judgment under uncertainty: Heuristics and biases (pp. 493-508). New York: Cambridge University Press.

Kasser, T., Cohn, S., Kanner, A. D., \& Ryan, R. M. (2007). Some costs of American corporate capitalism: A psychological exploration of value and goal conflicts. Psychological Inquiry, 18, 1-22.

Keller, M., \& Krettenaucr, T. (2007). Moralentwicklung im Kulturvergleich. In G. Trommsdorff \& H.-J. Kornadt (Hrsg.), Enzyklopädie der Psychologie: Themenbereich C. Theorie und Forschung: Serie VII. Kulturvergleichende Psychologie: Band 2. Erleben und Handeln im kulturellen Kontext (S. 521-555). Göttingen: Hogrefe.

Kogan, N., Lamm, H., \& Trommsdorff, G. (1972). Negotiation constraints in the risk taking domain: Effects of being observed by partners of higher or lower status. Journal of Personality and Social Psychology, 23, 143-156.

Kohn, M. L. (1984). The effects of social class on parental values and practices. In P. Voydanoff (Ed.), Work and family: Changing roles of men and women. Palo Alto, CA: Mayficld 
Kornadt, H.-J. (2007). Motivation im kulturellen Kontext. In G. Trommsdorff \& H.-J. Kornadt (Hrsg.), Enzyklopädie der Psychologie: Themenbereich C. Theorie und Forschung: Serie VI.l Kulturvergleichende Psychologie. Band 2: Erleben und Handeln im kulturellen Kontert (S. 283-376). Göttingen: Hogrefe.

Kornadt, H.-J. (in press). Quality of life and happiness - Transculturally comparable? In G. Szzéll, D. Elurig, U. Staroske, \& U. Széll (Eds.), Quality of life and working life in comparison. Bern: Peter Lang.

Künemund, H., \& Szydlik, M. (Hrsg.). (2009). Generationen: Multidiszi plinäre Pers pektiven. Wiesbaden: VS Verlag fŭr Sozialwissenschaften.

Lepsius, M. R. (1996). Lässt sich die deutsche Einheit durch Institutionalisierung herstellen? Festrede. In Jahrbuch der Heidelberger Akademie der Wissenschafien (S. 6166). Heidelberg.

Leyendecker, B., \& Schölmerich, A. (2007). Interdependente und independente Orientierungen in Kindheit und Jugend. In G. Trommsdorff \& H.-J. Kornadt (Hrsg.), Enzyklopädie der Psychologie: Themenbereich C. Theorie und Forschung: Serie VII. Kulturvergleichende Psychologie: Band 2. Erleben und Handeln im kulturellen Kontext (S. 557-597). Göttingen: Hogrefe.

Markus, H. R., \& Kitayama, S. (1991). Culture and the self: Implications for cognition, emotion, and motivation. Psychological Review, 98, 224-253.

Matsumoto, D. (2001). Culture and emotion. In D. Matsumoto (Ed.), The handbook of culture and psychology (pp. 171-194). New York: Oxford University Press.

Mayer, B. (2009). Adolescents' family models: A cross-cultural stucty. Unveröf fentlichte Dissertation, Universität Konstanz.

Mayer, B., \& Trommsdorff, G. (2009). Adolescents' value of children and their intentions to have children: A cross-cultural and multilevel analysis. Manuscript submitted for publication.

McCrea, S. M. (2008). Self-handicapping, excuse making, and counterfactual thinking: Consequences for self-esteem and future motivation. Journal of Personality and Social Psychology, 95, 274-292.

Mischel, W. (1996). From good intentions to willpower. In P. M. Gollwitzer \& J. A. Bargh (Eds.), The psychology of action: Linking cognition and motivation to behavior (pp. 197-218). New York: Guilford Press.

Mischel, W., \& Ayduk, Ö. (2004). Willpower in a cognitive-affective processing system: The dynamics of delay of gratification. In R. F. Baumeister \& K. D. Vohs (Eds.), Handbook of self-regulation: Research, theory, and applications (pp. 99-129). New York: Guilford Press.

Mishra, R. C., \& Dasen, P. R. (2005). Spatial language and cognitive development in India: An urban/rural comparison. In W. Friedlmeier, P. Chakkarath, \& B. Schwarz (Eds.), Culture and human development. The importance of cross-cultural research for the social sciences (pp. 153-179). Hove, UK: Psychology Press.

Müller, W. (2008). Schein oder Sein: Bildungsdisparitäten in der europäischen Statistik. Eine Illustration am Beispiel Deutschlands. Schmollers Jahrbuch. 128, 511-543.

Nauck, B. (2009). Value of children - eine spezielle Handlungstheorie des generativen Verhaltens und von Generationenbeziehungen im interkulturellen Vergleich. Kölner Zeitschrift für Soziologie und Sozialpsychologie, 53, 407-435.
Nave-Herz, R. (Hrsg.). (2002). Kontinuität und Wandel der Familie in Deutschland: Eine zeitgeschichtliche Analyse. Stuttgart: Lucius \& Lucius.

Nisbett, R. E. (2003). The geography of thought: How Asians and Westerners think differently ... and why. New York: Free Press.

Oyserman, D., Coon, H. M., \& Kemmelmeier, M. (2002). Rethinking individualism and collectivism: Evaluation of theoretical assumptions and meta-analyses. Psychological Bulletin, 128, 3-72.

Oyserman, D., \& Lee, S. W. S. (2008). Does culture influence what and how we think? Effects of priming individualism and collectivism. Psychological Bulletin, 134, 311 342.

PISA-Konsortium (Ed.). (2001). PISA 2000: Basiskompetenzen von Schülerinnen und Schülern im internationalen Vergleich. Opladen: Leske und Budrich.

PISA-Konsortium Deutschland (Ed.). (2008). PISA '06. PISA 2006 in Deurschland. Die Kompetenzen der Jugendlichen im dritten Ländervergleich. Münster: Waxmann.

Poortinga, Y. H. (2007). Dateninterpretation in der kulturvergleichenden Psychologie. In G. Tromunsdorff \& H.-J. Kornadt (Hrsg.), Enzyklopädie der Psychologie: Themenbereich C. Theorie und Forschung: Serie VII. Kulturvergleichende Psychologie Band 1. Theorien und Methoden in der kulturvergleichenden und kulturpsychologischen Forschung (S. 290-336). Göttingen: Hogrefe.

Popper, K. R. (1935). Die Logik der Forschung. Wien: Springer.

Rizzolatti, G., \& Sinigaglia, C. (2008). Empathie und Spiegelneurone. Die biologische Basis des Mitgefühls. Frankf urt am Main: Suhrkamp.

Röhl, H. C., Trommsdorff, G., Vainre, M., \& Heikamp, T. (2008). Are values related to acceptance of authority's autonomy? Unpublished project proposal, University of Konstanz.

Rothbaum, F., \& Trommsdorff, G. (2007). Do roots and wings oppose or complement one another? The socialization of autonomy and relatedness in cultural context. In J. E. Grusec \& P. Hastings (Eds.), The handbook of socialization (pp. 461-489). New York: The Guilford Press.

Ryan, R. M., \& Deci, E. L. (2000). Self-determination theory and the facilitation of intrinsic motivation, social development, and well-being. American Psychologist, 55, 68-78.

Scherer, K. R. (1997). The role of culture in emotion-antecedent appraisal. Journal of Personality and Social Psychology, 73, 902-922.

Schimmack, U., Schupp, J., \& Wagner, G. G. (2008). The influence of environment and personality on the affective and cognitive component of subjective well-being. Social Indicators Research, 89, 41-60.

Schwartz, S. H. (2004). Mapping and interpreting cultural differences around the world. In H. Vinken, J. Soeters \& P. Ester (Eds.), Comparing cultures: Dimensions of culture in a comparative pers pective (pp. 43-73). Leiden, The Netherlands: Brill Academic Publishers.

Schwartz, S. H. (2007). Cultural and individual value correlates of capitalism: A comparative analysis. Psychological Inquiry, 18, 52 57.

Schwarz, B., Albert, I., \& Trommsdorff, G. (2009). Intergenerational relations and life. satisfaction in mother-daughter dyads from Indonesia, China, and Germany. Manuscript submitted for publication. 
Schwarz, B., \& Trommsdorff, G. (2006). The relation between attachment and intergenerational support in Korea, China, and Gernany. Paper presented at the 18th International Congress of the International Association for Cross-Cultural Psychology (lACCP), Spetses, Greece.

Schwarz, B., Trommsdorff, G., Albert, I., \& Mayer, B. (2005). Adult parent-child relationship: Relationship quality, support, and reciprocity. Applied Psychology: An International Review, 54, 396-417.

Sherif, M. (1956). Experiments in group conflict. Scientific American. 195, 54-58.

Shiller, R., \& Akerlof, G. (2009). Animal spirits. How human psychology drives the economy: and why it matters for global ca pitalism. Princeton: Princeton University Press.

Silbereisen, R. K., Pinquart, M., \& Tomasik, M. J. (in press). Demands of social change and psychosocial adjustment: Results from the Jena study. In R. K. Silbereisen \& X. Chen (Eds.), Social change and human development: Concepts and results. London: Sage Publications.

Simon, H. A. (1979). Rational decision making in business organizations. American Economic Review, 69, 493-513

Smith, A. (1969). The theory of moral sentiments. New Rochelle, NY: Arlington House.

Smith, A. (1978). An inguiry into the nature and causes of the wealth of nation. München: Deutscher Taschenbuchverlag.

Song, Y., Lüer, G., \& Lass, U. (2007). Kulturvergleichende Wahmehmungs- und Kognitionsf orschung. In G. Trommsdorff \& H.-J. Kornadt (Hrsg.), Enzyklopädie der Psy chologie: Themenbereich C. Theorie und Forschung: Serie VII. Kulturvergleichende Psychologie: Band 2. Erleben und Handeln im kulturellen Kontext (S. 1-58). Göttingen: Hogrefe.

Statistisches Bundesamt (2008). Statistisches Jahrbuch für die Bundesrepublik Deutschland 2008. Wiesbaden: Statistisches Bundesamt.

Suchodoletz, A. v., Trommsdorff, G., Heikamp, T., Wieber, F., \& Gollwitzer, P. M. (in press). Transition to school: The role of preschooler's self-regulation. Leaming and Individual Differences.

Thibaut, J. W., \& Kelley, H. H. (1959). The social psychology of groups. New York: Wiley.

Tomasello, M. (2009). Cultural transmission: A view from chimpanzecs and human infants. In U. Schönpflug (Ed.), Cultural transmission: Developmental, psychological, social, and methodological aspects (pp. 33-48). New York: Cambridge University Press.

Trommsdorff, G. (Ed.). (1994). Psychologische Aspekte des sozio-politischen Wandels in Ostdeutschland. Berlin: de Gruyter.

Trommsdorff, G. (1995a). Identitätsprozesse im kulturellen Kontext und im sozialen Wandel. In H. Sahner (Hrsg.), Transformationsprozesse in Deutschland (S. 117148). Opladen: Leske und Budrich.

Trommsdorff, G. (1995b). Person-context relations as devclopmental conditions for $\mathrm{cm}$ pathy and prosocial action: A cross-cultural analysis. In T. A. Kindernann \& J. Valsiner (Eds.), Development of person-context relations (pp. 113-146). Hillsdalc. NJ: Erlbaum.
Trommsdorff, G. (2000). Psychological factors limiting institutional rehabilitation. In E. K. Scheuch \& D. Sciulli (Eds.), Societies, corporations, and the nation state (pp. 216-235). Leiden, The Netherlands: Brill Academic Publishers.

Trommsdorff, G. (2006a). Development of emotions as organized by culture. ISSBD Newsletter, 49, 1-4

Trommsdorff, G. (2006b). Parent-child relations over the life-span. A cross-cultural perspective. In K. H. Rubin \& O. B. Chung (Eds.), Parenting beliefs, behaviors, and parent-child relations. A cross-cultural perspective. New York: Psychology Press.

Trommsdorff, G. (2007a). Entwicklung im kulturellen Kontext. In G. Trommsdorff \& H. J. Kornadt (Hrsg.), Enzyklopädie der Psychologie: Themenbereich C Theorie und Forschung, Serie VII Kulturvergleichende Psychologie. Band 2: Kulturelle Determinanten des Erlebens und Verhaltens (S. 435-519). Göttingen: Hogrefe.

Trommsdorff, G. (2007b). Intentionality of action in cultural context. In J. Wassmann \& K. Stockhaus (Eds.), Experiencing new worlds. (pp. 58-77). New York: Berghahn.

Trommsdorff, G. (2008a). 25 Wellen des Sozio-Oekonomischen Panels (SOEP): Gewinn für interdisziplinäre Forschung. Vierteljahreshefıe zur Wirtschaftşforschung, 03/2008, 195-202.

Trommsdorff, G. (2008b). Entwicklungsbedingungen von Absichtlichkeit und ihrer Grenzen. Unveröffentlichter Projektantrag, Universität Konstanz.

Trommsdorff, G. (2009a). Intergenerational relations and cultural transmission. In U. Schönpflug (Ed.), Cultural transmission: Psychological, developmental, social, and methodological aspects (pp. 126-360). Cambridge: Cambridge University Press.

Trommsdorff, G. (2009b). A social change and a human devclopment perspective on the value of children. In S. Bekman \& A. Aksu-Koc (Eds.), Perspectives on human development, family and culture. Essays in honor of Cigdem Kagitcibasi (pp. 86-107). Cambridgc, UK: Cambridge University Press.

Trommsdorff, G. (2009c). Culture and development of self-regulation. Social and Personality Psychology Compass, 3, 1-15.

Trommsdorff, G. (in press). Indicators for quality of life and working life in Gernany and Japan: A culture-psychological perspective. In G. Széll, D. Ehrig, U. Staroske \& U. Széll (Eds.), Quality of life and working life in comparison. Bem: Peter Lang.

Trommsdorff, G., \& Albert, I. (2009). Kulturvergleich vor Beziehungsqualität in Mehrgenerationenfamilien aus psychologischer Sicht. In H. Künemund \& M. Szydlik (Hrsg.), Generationen - Multidisziplinäre Perspektiven (S. 119-134). Wiesbaden: VS Verlag für Sozialwissenschaften.

Trommsdorff, G., \& Chakkarath, P. (mit Heller, P.) (1996). Kindheit im Transfonnationsprozeß. In S. E. Hormuth, W. R. Heinz, H.-J. Komadt, H. Sydow, \& G. Trommsdorff (Hrsg.), Individuelle Entwicklung, Bildung und Berufsverläufe. Berichte zum sozialen und politischen Wandel in Ostdeutschland der KSPW, Bd. 4 (S. 11-77). Opladen: Leske und Budrich.

Trommsdorff, G., \& Cole, P. M. (in press). Emotion, self-regulation, and social behaviour in cultural contexts. In X. Chen \& K. H. Rubin (Eds.), Socioemotional development in cultural context. New York: Guilford.

Trommsdorff, G., \& Friedlmeier, W. (1999). Emotionale Entwicklung im Kulturvergleich. In W. Friedlmeier \& M. Holodynski (Hrsg.), Emotionale Entwicklung: Funk- 
tion, Regulation und soziokultureller Kontext von Emotionen (S. 275-293). Heidelberg: Spektrum

Trommsdorff, G., \& Friedlmeier, W. (in press). Preschool girls' distress and mothers' sensitivity in Japan and Germany. European Journal of Developmental Psychology.

Trommsdorft; G., Friedlmeier, W., \& Mayer, B. (2007). Sympathy, distress, and prosocial behavior of preschool children in four cultures. International Journal of Behavioral Development, 31, 284-293.

Trommsdorff, G., Kim, U., \& Nauck, B. E. (2005). Factors influencing value of children and intergenerational relations in times of social change: Analyses from psychological and socio-cultural perspectives [Special Issue]. Applied Psychology: An International Review, 54, 313-316.

Trommsdorff, G., \& Komadt, H.-J. (1995). Prosocial and antisocial motivation of adolescents in East and West Gemnany. In J. Youniss (Ed.), After the wall: Family adaptations in East and West Germany. New directions for child development, No. 70 (pp. 39-56). San Francisco: Jossey-Bass.

Trommsdorff, G., \& Kornadt, H.-J. (Hrsg.). (2007a). Enzyklopädie der Psychologie Themenbereich C. Theorie und Forschung: Serie VII. Kulturvergleichende Psychologie: Band I. Theorien und Methoden in der kalturvergleichenden und kulturpsychologischen Forschung. Göttingen: Hogrefe.

Trommsdorff, G., \& Komadt, H.-J. (Hrsg.). (2007b). Enzyklopädie der Psychologie Themenbereich C. Theorie und Forschung: Serie VII. Kulturvergleichende Psychologie: Band 2. Erleben und Handeln im kulturellen Kontext. Göttingen: Hogrefe.

Trommsdorff; G., \& Kornadt, H. J. (Hrsg.). (2007c). Enzyklopädie der Psychologie: Themenbereich C. Theorie und Forschung: Serie VII. Kulturvergleichende Psychologie: Band 3. Anwendungsfelder der kulturvergleichenden Psychologie. Göttingen: Hogrefe.

Trommsdorff, G., Kornadt, H.-J., \& Hessel-Scherf, M. (1998). Soziale Motivation ostund westdeutscher Kinder. In H. Oswald (Hrsg.), Sozialisation und Entwicklung in den neuen Bundesländern: Ergebnisse empirischer Längsschnittstudien (S. 121136). Weinheim: Juventa.

Trommsdorff, G., \& Mayer, B. (2005). Kulturvergleichende Ansätze. In H. Weber \& T. Rammsayer (Hrsg.), Handbuch der Persönlichkeitspsychologie und Differentiellen Psychologie. Handbuch der Psychologie (S. 220-228). Göttingen: Hogrefe.

Trommsdorff, G., \& Nauck, B. (Eds.). (2005). The value of children in cross-cultural perspective. Case studies from eight societies. Lengerich: Pabst Science.

Trommsdorff, G., \& Nauck, B. (2006). Demographic changes and parent-child relationship. Parenting: Science and Practice, 6, 343-360.

Trommsdorff, G., \& Nauck, B. (submitted). Introduction to the special section: Value of Children - a concept for the better understanding of cross-cultural variations of fertility behavior and intergenerational relationships. Journal of Cross-Cultural Psychology.

Trommsdorff, G., \& Rothbaum, F. (2008). Development of emotion regulation in cultural context. In S. Ismer, S. Jung, S. Kronast, C. v. Scheve, \& M. Vandekerckhove (Eds.), Regulating emotions: Social necessity and biological inheritance. (pp. 85120). London: Blackwell.
Van de Vijver, F. J. R. (2007). Methodologische und methodische Probleme des Kulturvergleichs. In G. Trommsdorff \& H.-J. Komadt (Hrsg.), En:yklopädie der Psychologie: Themenbereich C. Theorie und Forschung: Serie VIl. Kulturvergleichende Psychologie: Band 1. Theorien und Methoden der kulturvergleichenden Psychologie (S. 338-382). Göttingen: Hogrefe

Van de Vijver, F. J. R., Hemert, D. A., \& Poortinga, Y. H. (Eds.). (2008). Multilevel analysis of individuals and culture. New York: Erlbaum.

Van de Vijver, F. J. R., \& Leung, K. (1997). Methods and data anal ysis for cross"cultural research. London: Sage.

Wagner, G. G., Spieß, C. K., \& Schupp, J. (2008). Die verhaltenswissenschaf tliche Weiterentwicklung des Erhebungsprogramms des SOEP. Vierteljahreshefte zur Wirtschafts forschung, 03/2008, 63-76.

Weber, M. (1988). Gesammelte Aufsätze zur Religionssoziologie (9. Aufl.). Tübingen: Mohr.

Wundt, W. (1900-1920). Völkerpsychologie: Eine Untersuchung der Entwicklungsgesetze von Sprache, Mythus und Sitte (10 Bde.). Leipzig: Engelmann \& Kröner.

Zimbardo, P. G. (2007). The Lucifer effect: Understanding how good people turn evil. New York: Random House. 